\title{
B cell-derived IL-4 acts on podocytes to induce proteinuria and foot process effacement
}

\author{
Alfred H.J. Kim, ${ }^{1}$ Jun-Jae Chung, ${ }^{2}$ Shreeram Akilesh, ${ }^{2}$ Ania Koziell, ${ }^{3}$ Sanjay Jain, ${ }^{4}$ Jeffrey B. Hodgin, ${ }^{5}$ \\ Mark J. Miller, ${ }^{2}$ Thaddeus S. Stappenbeck, ${ }^{2}$ Jeffrey H. Miner, ${ }^{4}$ and Andrey S. Shaw ${ }^{2,6}$ \\ 'Division of Rheumatology, Department of Internal Medicine, and 2Division of Immunobiology, Department of Pathology \\ and Immunology, Washington University School of Medicine, St. Louis, Missouri, USA. ${ }^{3}$ Department of Experimental \\ Immunobiology, Division of Transplantation Immunology and Mucosal Biology, King's College London and Department \\ of Paediatric Nephrology, Evelina Children's Hospital, London, United Kingdom. ${ }^{4}$ Renal Division, Washington University \\ School of Medicine, St. Louis, Missouri, USA. '5 Department of Pathology, University of Michigan, Ann Arbor, Michigan, \\ USA. ${ }^{6}$ Howard Hughes Medical Institute, Washington University School of Medicine, St. Louis, Missouri, USA.
}

The efficacy of B cell depletion therapies in diseases such as nephrotic syndrome and rheumatoid arthritis suggests a broader role in B cells in human disease than previously recognized. In some of these diseases, such as the minimal change disease subtype of nephrotic syndrome, pathogenic antibodies and immune complexes are not involved. We hypothesized that B cells, activated in the kidney, might produce cytokines capable of directly inducing cell injury and proteinuria. To directly test our hypothesis, we targeted a model antigen to the kidney glomerulus and showed that transfer of antigen-specific $B$ cells could induce glomerular injury and proteinuria. This effect was mediated by IL-4, as transfer of IL-4-deficient B cells did not induce proteinuria. Overexpression of IL-4 in mice was sufficient to induce kidney injury and proteinuria and could be attenuated by JAK kinase inhibitors. Since IL-4 is a specific activator of STAT6, we analyzed kidney biopsies and demonstrated STAT6 activation in up to 1 of 3 of minimal change disease patients, suggesting IL-4 or IL-13 exposure in these patients. These data suggest that the role of B cells in nephrotic syndrome could be mediated by cytokines.

Conflict of interest: A.H.J. Kim received advisory board income from Exagen Diagnostics Inc. and research funding from Kypha Inc. J.H. Miner has received licensing fees from Cenentech and Eli Lilly, research funding from $F$. Hoffmann-La Roche, and advisory board income from Regulus Therapeutics. J.J. Chung and A.S. Shaw are employees of Cenentech.

Submitted: March 12, 2015 Accepted: October 5, 2017 Published: November 2, 2017

\section{Reference information:} JCI Insight. 2017;2(21):e81836. https://doi.org/10.1172/jici. insight.81836

\section{Introduction}

B cell depletion therapies (BCDTs), such as rituximab, are efficacious in numerous immune-mediated diseases, including rheumatoid arthritis and multiple sclerosis (1). Current BCDTs target CD20 and eliminate $\mathrm{B}$ cells but not antibody-producing plasma cells because they do not express CD20 (2). Indeed, most autoantibodies remain present after depletion despite clinical response (3), suggesting that B cells may contribute to tissue injury in an antibody-independent manner.

Since the first reports in 2004 of the successful treatment of steroid-sensitive nephrotic syndromes (SSNS), such as minimal change disease (MCD) and focal segmental glomerulosclerosis (FSGS), using rituximab (4-6), there are now over 200 publications in the literature. The majority of these are anecdotal reports and small uncontrolled studies, but the consensus today is that BCDTs are likely efficacious for steroid-sensitive, relapsing forms of disease. By definition, autoreactive antibodies and immune complexes are absent in MCD and FSGS (7). Current therapies are based on the idea that immune dysfunction, potentially involving $\mathrm{T}$ cells, is involved in the pathogenesis of these diseases, as first proposed by Shalhoub (8). This idea is supported by the responsiveness of most MCD/FSGS patients to immunosuppressive therapies. Furthermore, the clinical observation that some patients with nephrotic syndrome respond to plasmapheresis suggests that a soluble, circulating factor may be responsible $(9,10)$. The efficacy of BCDTs supports an immune origin of disease for some nephrotic patients. However, a specific mechanism to explain disease pathogenesis is still lacking.

Given the absence of pathogenic antibodies in these diseases, a role for B cells would need to be antibody independent. In recent years, antibody-independent functions have been ascribed to B cells. Mouse models of systemic lupus erythematosus (SLE) demonstrate that B cells are as important as 
A

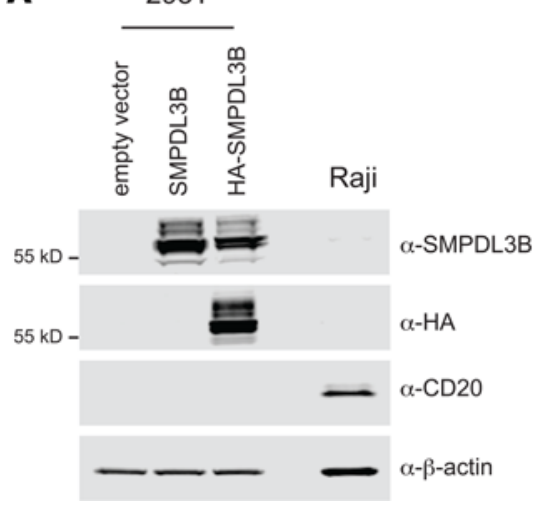

B

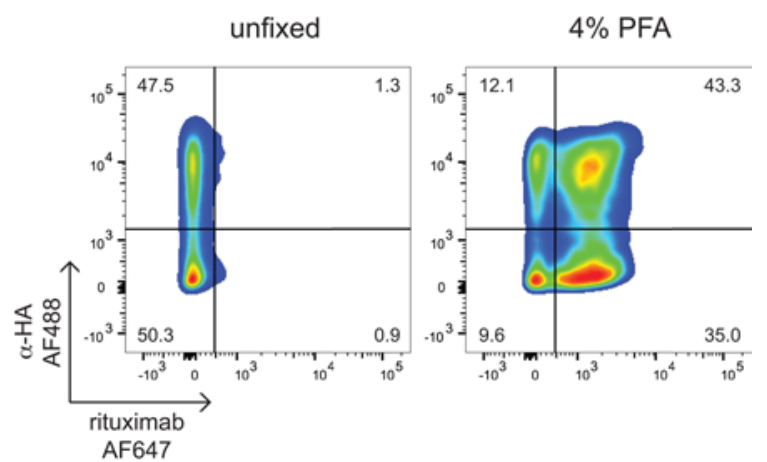

Figure 1. Rituximab does not bind SMPDL3B in nonfixed samples. (A) Western blot of transfected 293T and Raji cells. Whole-cell lysates were subjected to SDS-PAGE, followed by immunoblotting with antibodies as indicated. $\beta$-Actin was used as loading control. (B) Rituximab does not bind to SMPDL3B in nonfixed cells. Nonfixed or PFA-fixed 293T cells expressing HA-SMPDL3B were stained with $\alpha$-HA-AF488 and rituximab-AF647 and analyzed by flow cytometry. Data are representative of 3 independent experiments.

antigen-presenting cells for autoreactive T cells (11). B cells can also make cytokines that can either promote or inhibit inflammation $(12,13)$. Cytokines produced by B cells are required for the development of lymph node and ectopic lymphoid follicles, can amplify $\mathrm{T}$ cell responses, and can suppress arthritis, colitis, and experimental autoimmune encephalitis in mice $(14,15)$.

The cardinal pathologic feature of nephrotic diseases is foot process effacement of the glomerular podocyte. The glomerulus houses the glomerular filtration barrier, where a highly differentiated epithelial cell known as the podocyte forms the final cellular barrier for the filtration of blood and generation of urine. Podocytes have a specialized architecture characterized by interdigitating cellular arborizations (foot processes) that form a mesh-like structure on the urinary aspect of the glomerular capillaries. In all cases of active proteinuria, podocytes exhibit a simplification and flattening/retraction of the podocyte foot processes, a finding termed foot process effacement. Given the lack of cellular inflammation or immune complexes in MCD and FSGS, the likeliest scenario for an immunologic cause would be a factor secreted by an immune cell.

In this study, we directly tested whether cytokines produced by B cells could induce podocyte foot process effacement and proteinuria. Using an in vitro assay of podocyte injury, we identified IL-4 as a cytokine capable of stimulating membrane ruffling of podocytes and detachment of podocytes from the basement membrane in vitro. Using a method of in vivo gene delivery, we confirmed that IL-4 is sufficient to induce foot process effacement and proteinuria when overexpressed in mice. To determine whether B cells activated locally in the kidney could make enough IL-4 to induce foot process effacement, we planted a B cell antigen on the glomerular basement membrane (GBM) and showed that antigen-specific B cells could induce foot process effacement in an IL-4-dependent fashion. Finally, we found evidence of IL-4 receptor signaling in the kidneys in some patients with MCD. Taken together, these findings reveal a mechanism for the efficacy of anti-B cell therapies in the treatment of steroid-sensitive nephrotic diseases. These results also suggest that IL-4 is a potential nephrotic factor and therapeutic target.

\section{Results}

Rituximab does not bind SMPDL3B in nonfixed cells. It was recently proposed that the effect of rituximab on nephrotic syndrome is not due to B cells but is instead mediated by its off-target binding to sphingomyelin-phosphodiesterase-acid-like-3b (SMPDL3B) expressed on podocytes (16). To confirm the binding of SMPDL3B by rituximab, we overexpressed HA-tagged SMPDL3B in 293T cells that do not endogenously express either CD20 or SMPDL3B. Expression and membrane localization of HA-SMPDL3B were confirmed by immunoblotting and immunofluorescence with HA and SMPDL3B antibodies (Figure 1A and Supplemental Figure 1, $\mathrm{A}$ and $\mathrm{B}$; see complete unedited blots in the supplemental material; supplemental material available online with this article; https://doi.org/10.1172/jci.insight.81836DS1). Modeling suggested that the N-terminal HA-tag is spatially separated from the SMPDL3B epitope $\left({ }^{156} \mathrm{ELWKPW}^{161}\right)$ reported to be recognized by rituximab (Supplemental Figure 1C). 
A

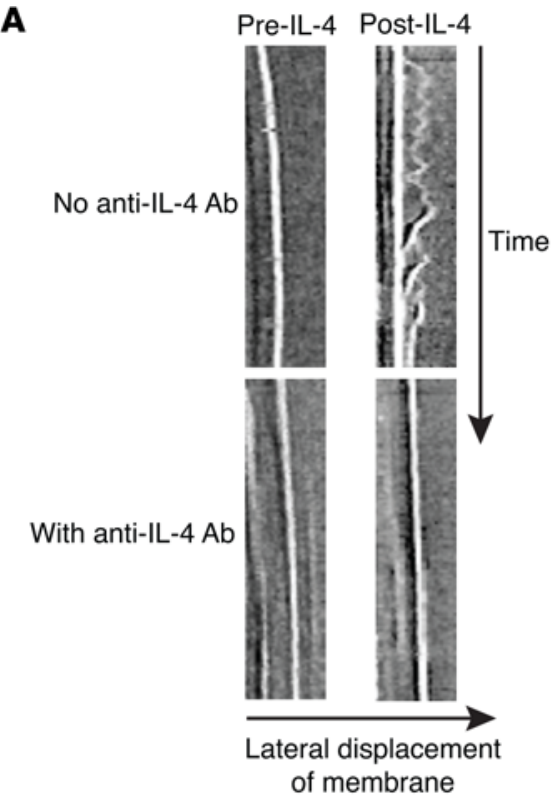

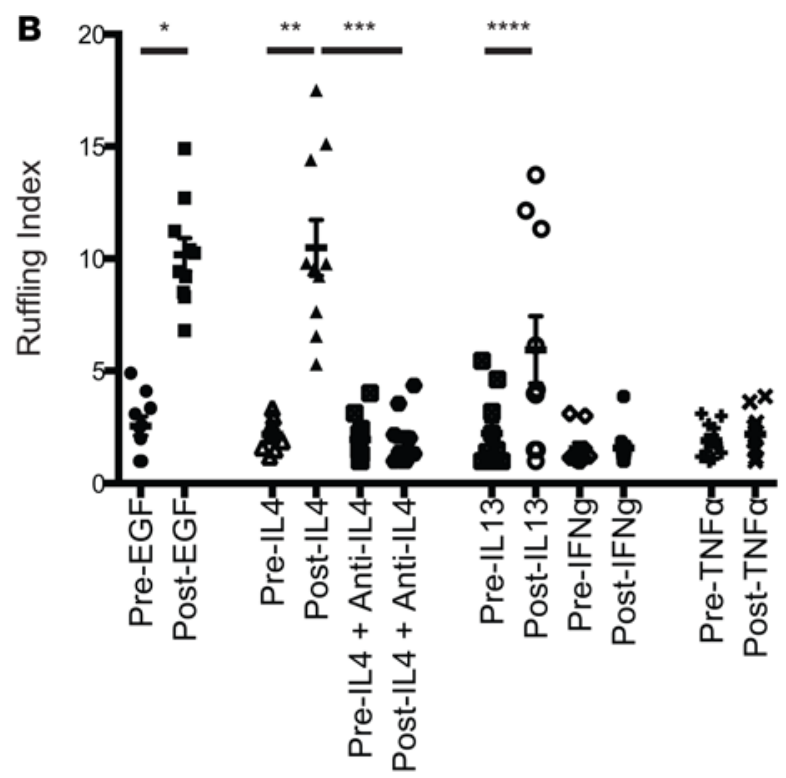

C

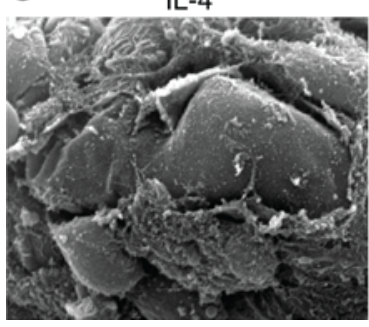

IL-4 + Anti-IL-4 Ab

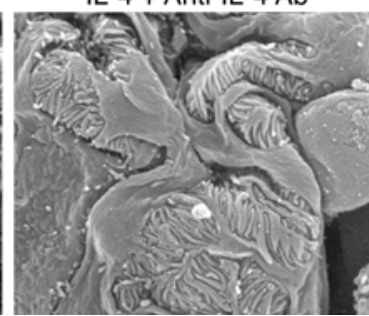

EGF

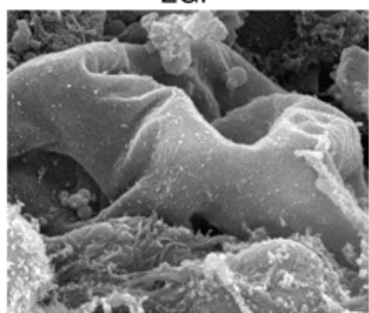

TNF-a

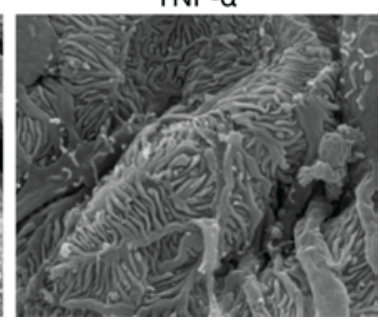

Figure 2. IL-4 induces podocyte membrane ruffling and widespread foot process retraction. (A) Differential interference contrast live-cell imaging was used to obtain kymographs from individual differentiated cultured murine podocytes. Each cell was imaged individually from separate podocyte cultures. Representative kymographs (time $=20$ minutes) before (left column) and after (right column) the addition of IL-4 (10 ng/ml, top row) or IL-4 with anti-IL-4 antibody $(5 \mu \mathrm{g} / \mathrm{ml}$, bottom row) are shown. IL-4 caused pronounced membrane ruffling that was abrogated by anti-IL-4 treatment (also see Supplemental Videos 1 and 2). Original magnification, $x \sim 1,100$. (B) Kymograph analysis was used to quantitate actin spike lengths (ruffling index) of 5 locations per cultured podocyte for the cytokines tested (IL-13: $10 \mathrm{ng} / \mathrm{ml}$, IFN- $\gamma: 100 \mathrm{ng} / \mathrm{ml}$, TNF- $\alpha: 10 \mathrm{ng} / \mathrm{ml}$ ). IL-4 and IL-13 have enhanced membrane ruffling dynamics. IL-4 promoted an equivalent level of ruffling as a known activator of Rac, EGF ( $20 \mathrm{ng} / \mathrm{ml})$, which was reversed with anti-IL-4 antibody. Each symbol represents the average relative cell membrane displacement over time at 5 regions of a single cell. Each cell mean \pm SD of 3 experiments, with 3-4 cells/ experiment (total of 10 cells analyzed/group). (C) Representative scanning electron microscopy images of glomeruli from minced renal cortices incubated ex vivo for 20 minutes with IL-4 (10 ng/ml), IL-4 with anti-IL-4 antibody $(5 \mu \mathrm{g} / \mathrm{ml})$, TNF- $\alpha(10 \mathrm{ng} / \mathrm{ml})$, or positive control EGF $(20 \mathrm{ng} / \mathrm{ml})$. IL-4 treatment induced massive foot process retractions similar to that induced by EGF. TNF- $\alpha$ and IL-4 plus anti-IL-4 did not alter foot process morphology, consistent with the membrane ruffling data. Scale bar: $20 \mu \mathrm{m}$. ${ }^{*} P<0.001,{ }^{* *} P<0.005,{ }^{* *} P<0.004,{ }^{* * * *} P<0.03$ by 1 -way ANOVA with Bonferroni correction.

To mimic the in vivo setting, we incubated nonfixed cells with rituximab and an anti-HA tag antibody. In 293T cells expressing HA-SMPDL3B, staining was detected with an anti-HA antibody but not with rituximab (Figure 1B and Supplemental Figure 2, A and B). Notably, when cells were fixed with paraformaldehyde (PFA) prior to antibody staining, we observed marked binding of rituximab to cells, regardless of SMPDL3B expression (Figure 1B and Supplemental Figure 2, A and B). Similar results were obtained with HeLa cells overexpressing HA-SMPDL3B (Supplemental Figure 3, A and B; see complete unedited blots in the supplemental material). Binding of rituximab to CD20 was confirmed by staining Raji cells (Supplemental Figure 1D). These results suggest that rituximab does not bind to SMPDL3B in vivo and that the binding previously reported is not specific. This suggests that the efficacy of rituximab in nephrotic kidney diseases is not through its ability to bind and directly modulate podocytes. We therefore considered antibody-independent B cell functions.

IL-4 and IL-13 induced membrane ruffing in cultured podocytes and foot process retraction in kidney fragments in vitro. B cells secrete a diverse array of cytokines upon activation, including IFN- $\gamma$, TNF- $\alpha$, IL-4, and IL-13 (13, 1720). Conversely, podocytes express cytokine receptors, including receptors for IFN- $\gamma$, TNF- $\alpha$, IL- 4 , and IL-13 (21-23). Therefore, we tested individual cytokines for their ability to induce foot process effacement in vitro. 
A

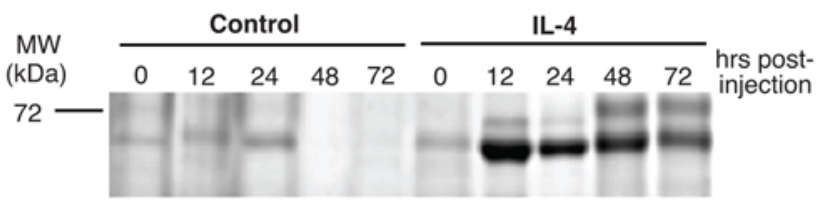

\section{B}

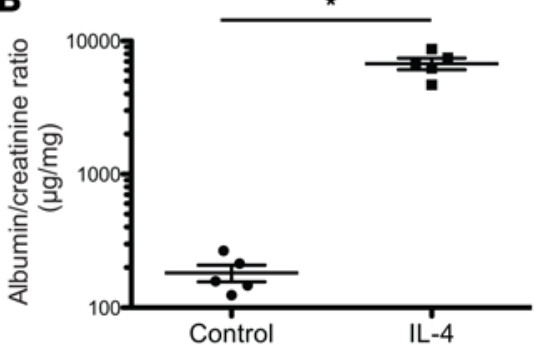

D

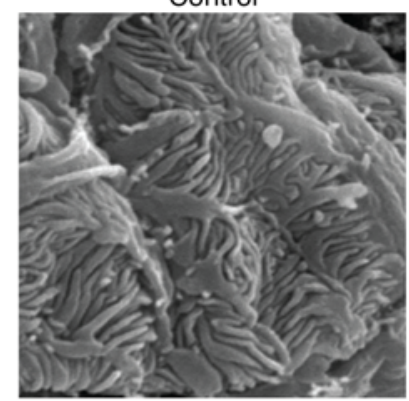

E

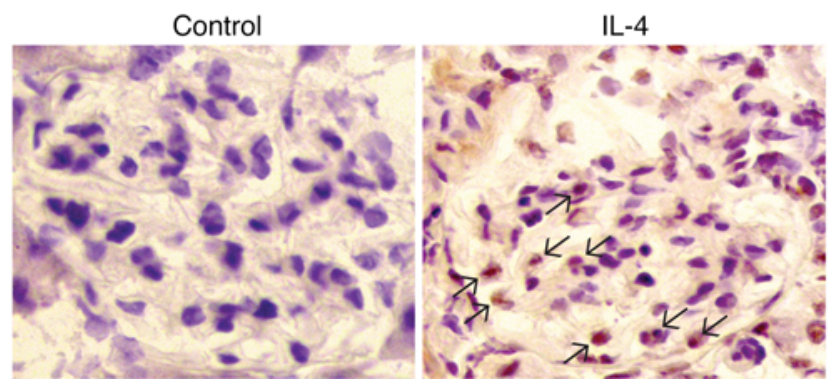

C

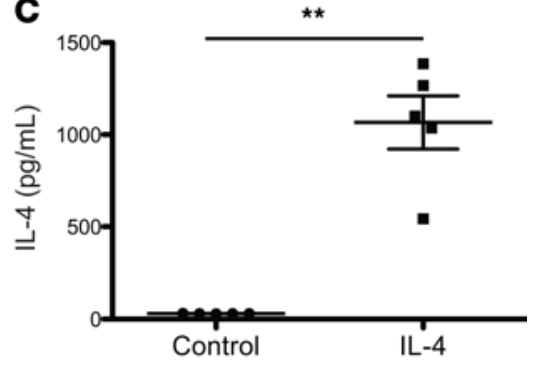

IL-4

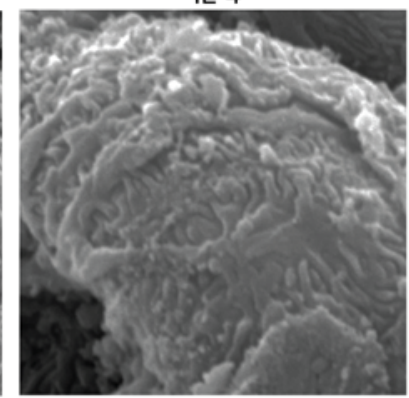

IL-4

Figure 3. Mice treated with a plasmid encoding IL-4 exhibited proteinuria, foot process abnormalities, and STAT6 activation in glomeruli. (A) 129X1/Sv] mice were hydrodynamically injected with either IL-4 piggyBac plus transposase vectors to induce IL-4 expression (IL-4 treated) or empty piggyBac plus transposase vectors (control). Representative Coomassie blue-stained SDS-PAGE. (B) Spot albumin/creatinine ratios of urine from control or IL-4-treated mice demonstrated proteinuria with IL-4 expression. Urine was collected 12 hours after plasmid injection. (C) Serum IL-4 ELISA confirmed elevated expression of IL-4 in IL-4 gene-treated mice. Symbols represent individual mice, and bars represent the geographic mean in $\boldsymbol{B}$ and mean in C. Mean $\pm \mathrm{SD}$ of 3 experiments, with total of 5 mice/group. ${ }^{*} P<0.006$, ${ }^{*} P<0.001$ by 2 -tailed Mann-Whitney. (D) Representative scanning electron microscopy (scale bar: 1 $\mu \mathrm{m})$ revealed foot process retraction with focal foot process effacement in IL-4-treated mice compared with control. (E) Representative immunohistochemical analysis of glomerular PSTAT6 expression reveals substantial STAT6 phosphorylation and nuclear translocation in IL-4-treated mice (black arrows) compared with control. Original magnification, $\times 400$. Data are representative of 4 independent experiments.

We have proposed that the activation of the small GTPase Rac is the cell biological correlate for foot process effacement in vivo $(24,25)$. Rac is a member of the Rho family of small $G$ proteins, which also includes Cdc42 and Rho. Rac activation stimulates the formation of actin cytoskeletal structures that are associated with cell migration and membrane ruffling (26). Rac activation in podocytes acutely induces foot process effacement and proteinuria in mice $(24,25)$.

Cytokines were added to cultured murine podocytes, and differential interference contrast live-cell imaging of individual cells in separate culture dishes was used to evaluate the response. Membrane ruffling was used as a surrogate for Rac activation, and kymograph analysis was used to quantitate membrane ruffling before and after addition of the cytokine $(25,27)$. IL-4 and, to a lesser extent, IL-13 induced membrane ruffling, while TNF- $\alpha$ and IFN- $\gamma$ had no effect on membrane ruffling (Figure 2, A and B, and Supplemental Videos 1 and 2). IL-4 stimulated a similar degree of ruffling as EGF, a known activator of Rac (28). The addition of a neutralizing IL-4 antibody abrogated IL-4-induced membrane ruffling (Figure 2B), indicating that the effect was IL-4 specific.

Since cultured podocytes do not form foot processes, we minced murine renal cortices where the glomeruli are located and treated these fragments with cytokines to evaluate for abnormalities in foot process morphology. Using scanning electron microscopy, we found that IL-4 induced severe foot process retractions, podocyte detachment, and exposure of the glomerular capillary wall, while TNF- $\alpha$ did not (Figure 2C). The changes induced by IL- 4 were comparable to those induced by EGF and were blocked with anti-IL-4 antibody (Figure $2 \mathrm{C}$ ). These data confirmed the ability of IL-4 to directly induce podocyte damage in ex vivo renal cortex. 

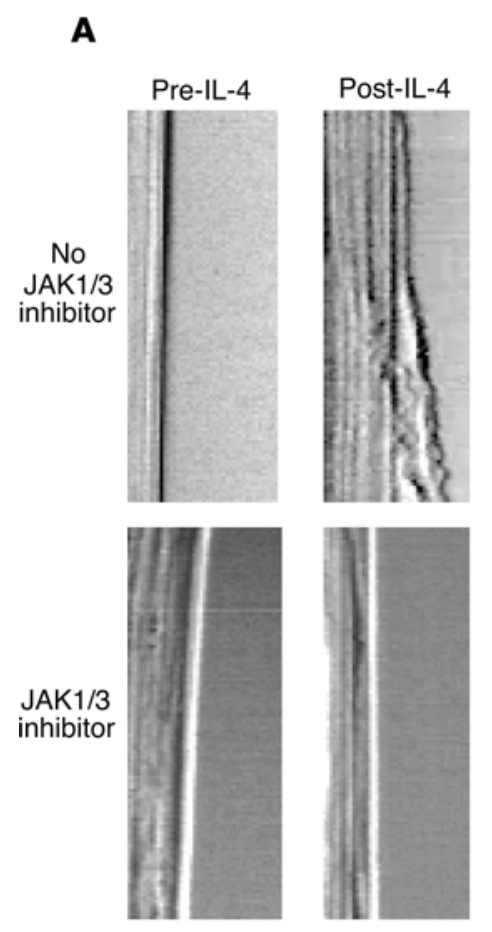
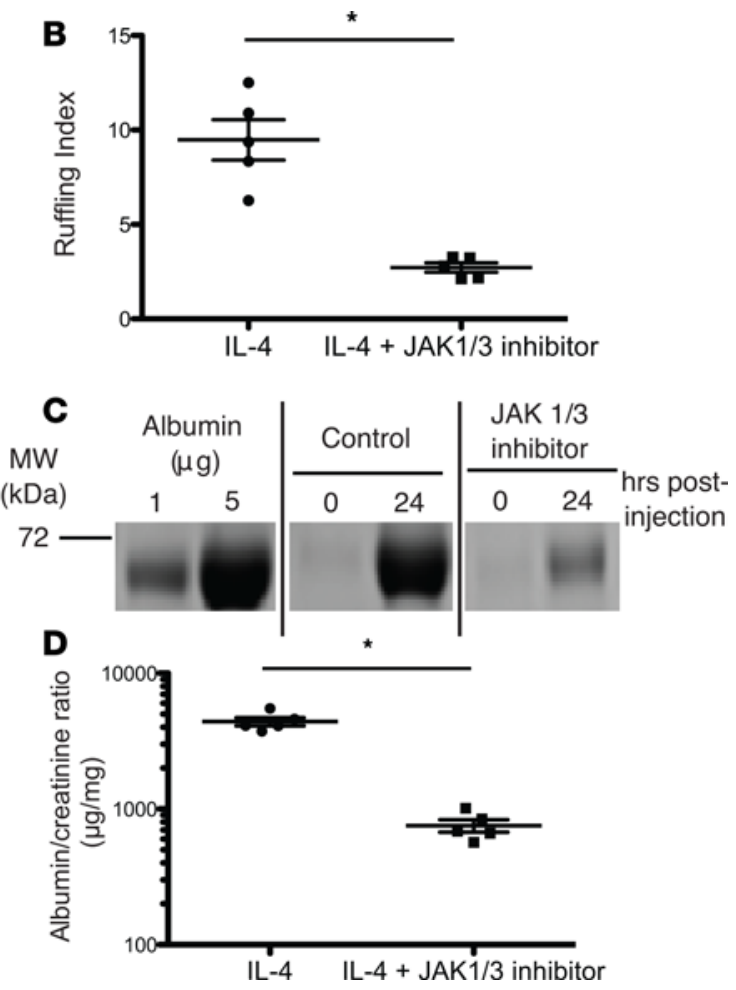

Figure 4. Inhibition of IL-4 signaling with JAK1/3 inhibitor abrogated ruffling and proteinuria in IL-4-treated mice. (A) Representative kymographs obtained by membrane ruffling and (B) quantification, as performed in Figure 1, from cultured podocytes treated with the JAK1/3 inhibitor tofacitinib (100 $\mathrm{nM}$, bottom row), which significantly attenuated IL-4-induced membrane ruffling compared with negative control (DMSO). Each symbol represents the average relative cell membrane displacement over time at 5 regions of a single cell. Mean \pm SD of 2 experiments, with 2-3 cells/experiment (total of 5 cells analyzed/group). (C) Coomassie blue-stained SDS-PAGE and (D) spot albumin/creatinine ratios of urine from IL-4-treated mice treated with JAK1/3 inhibition, which significantly reduced IL-4-induced proteinuria. Urine was collected 24 hours after plasmid administration. Symbols represent individual mice, and bars represent the mean. Mean \pm SEM of 2 experiments, with total of 5 mice/group. ${ }^{*} P<0.008$ by 2 -tailed Mann-Whitney.

IL-4 overexpression induces proteinuria and IL-4 signaling in the glomerulus in vivo, which is reduced with JAK1/3 inhibition. To evaluate the role of IL-4 in vivo, we expressed IL-4 in mice by injecting a plasmid based on a transposon expression system using hydrodynamic injection (29-31). In this system, the gene of interest integrates into the genome in a site-specific fashion, primarily in hepatocytes, allowing for efficient and high-level expression of the introduced gene (32). Delivery of the IL-4 gene induced marked proteinuria (Figure 3, $\mathrm{A}$ and $\mathrm{B}$; see complete unedited blots in the supplemental material) with a severity that directly correlated with serum levels of IL-4 (Figure 3C). No proteinuria was seen when a control targeting vector was administered. Scanning electron microscopy performed on kidneys 3 days after plasmid administration demonstrated blunted foot processes with scattered areas of effacement (Figure 3D). In comparison, the foot processes of podocytes from control mice were normal. These data show that high serum levels of IL-4 via its overexpression in the liver can directly induce podocyte foot process abnormalities and proteinuria in vivo.

IL-4 signals through the IL-4 receptor (IL-4R $\alpha$ and common- $\gamma$ chain) to activate the tyrosine kinases JAK1 and JAK3 and subsequently the transcription factor STAT6 (33). Kidney sections obtained from mice 24 hours following mock or IL-4 plasmid administration were stained for 6 (pSTAT6) using immunohistochemistry (Figure 3E). IL-4 treatment strongly induced pSTAT6 within podocytes and other glomerular cells, with little to no staining detected in the glomeruli of control mice. Based on the activation of STAT6, this supports the idea that podocytes can respond to IL-4 in vivo.

Since IL-4 utilizes the JAK1 and JAK3 kinases for signaling, we tested if JAK1/3 inhibition could block IL-4-induced membrane ruffling in podocytes in vitro and the development of proteinuria in vivo. A small-molecule inhibitor of JAK1 and JAK3, tofacitinib, is approved for the treatment of rheumatoid arthritis (34). Pretreatment of cultured podocytes with the inhibitor significantly decreased membrane ruffling induced by IL-4 (Figure 4, A and B). To assess its effects in vivo, mice were treated with the inhibitor twice daily, starting 1 day prior to IL-4 gene delivery. Treatment with the JAK inhibitor 
A $\underset{\mathrm{HEL}}{\text { Monomic }} \stackrel{\text { Biotinylated }}{\mathrm{HEL}}$
B

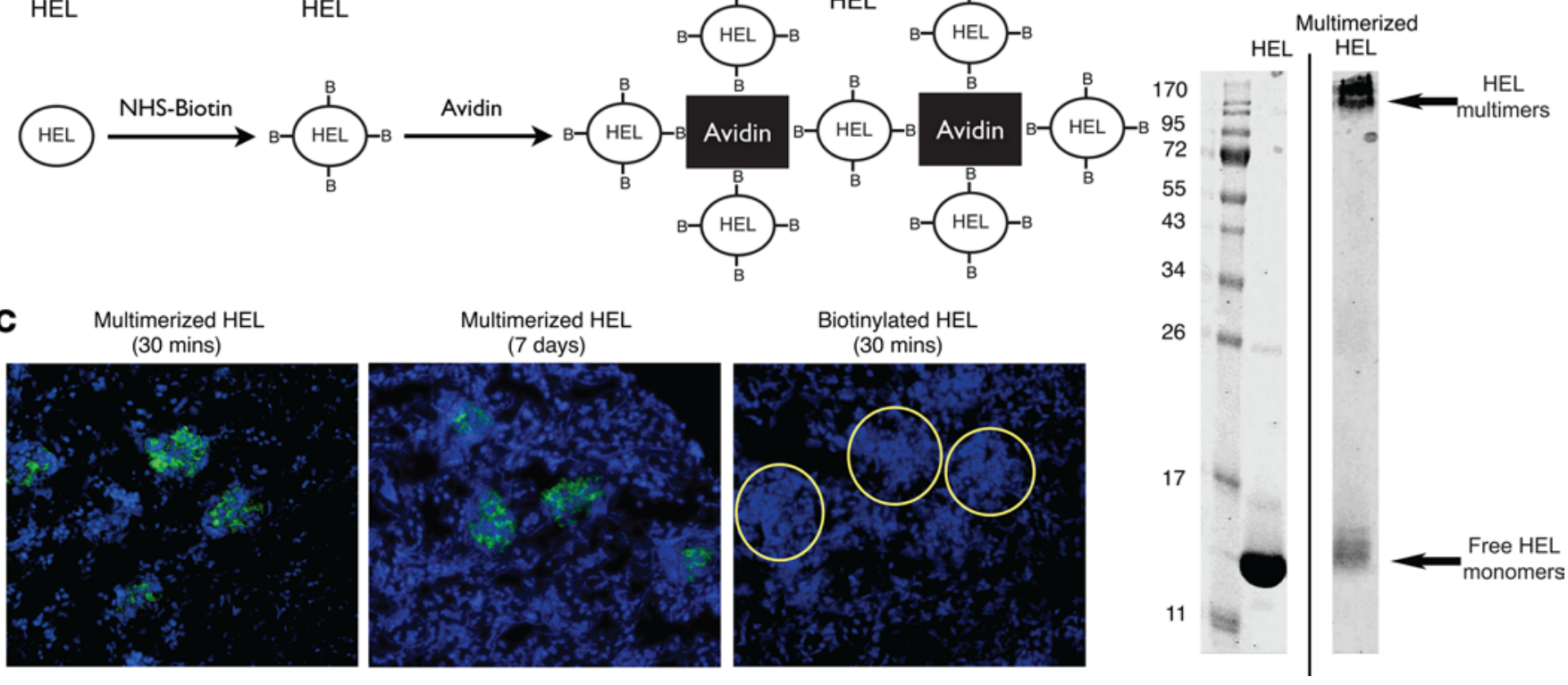

Figure 5. Multimerized hen egg lysozyme embedded within the glomerular basement membrane following i.v. injection. (A) The production of multimerized hen egg lysozyme (HEL). Monomeric HEL was biotinylated and then complexed with avidin to form multimers. (B) Representative Coomassie bluestained SDS-PACE of monomeric and multimerized HEL demonstrated multimerized complexes of at least $120 \mathrm{kDa}$ were formed. (C) Immunofluorescence microscopy images (green = HEL complex, blue = DAPI) of frozen sections from kidneys of mice injected with multimerized HEL (left: 30 minutes after injection, center: 7 days after injection) or monomeric HEL (right: 30 minutes after injection) and stained with anti-HEL antibody. Monomeric HEL was not visible in glomeruli, while multimerized HEL was found in the glomeruli up to 7 days after injection. Glomeruli are outlined in circles. Original magnification, $\times 20$. Data are representative of 3 independent experiments.

significantly attenuated the proteinuria induced by IL-4 (Figure 4, C and D; see complete unedited blots in the supplemental material). Thus, JAK1/3 inhibition can block the effects of IL- 4 on foot process effacement and proteinuria.

Generation of a B cell-dependent in vivo model of proteinuria. We next wondered if B cells could be activated in the glomerulus and whether they could produce sufficient IL- 4 to induce podocyte foot process effacement and proteinuria in vivo. To test this hypothesis, we targeted a B cell antigen to the glomerular capillary wall, followed by an infusion of antigen-specific B cells. We chose the soluble antigen hen egg lysozyme (HEL), as a B cell receptor-specific transgenic mouse exists for it (35). HEL has a high negative $\log$ dissociation constant $\left(\mathrm{p} K_{i}\right)(\sim 12)$ that allows it to enter the negatively charged glomerular filtration barrier, but its small size $(14 \mathrm{kDa})$ allowed it to be freely filtered through the GBM. To trap HEL within the glomerular capillary wall, we conjugated it to biotin, allowing us to produce larger tetrameric aggregates using avidin (Figure 5A). The HEL multimers had a molecular weight greater than $120 \mathrm{kDa}$ (Figure 5B; see complete unedited blots in the supplemental material), well above the size exclusion limit ( $60-70 \mathrm{kDa})$ of the glomerular filtration barrier. I.v. injection of avidin/biotin/HEL complexes resulted in trapping of multimers in the GBM within 30 minutes (Figure 5C) and was stable up to a week after injection. HEL multimers were also detected in the liver and spleen (data not shown).

We next tested whether adoptive transfer of polarized antigen-specific B cells could induce proteinuria. B cells were polarized into B effector 2 ( $\mathrm{Be} 2$ ) cells by activating them in the presence of Th2-polarized cells (Figure 6A); then they were injected into mice i.v. $(13,36)$. Within 24 hours, mice became proteinuric, as detected qualitatively using SDS-PAGE of urine samples or quantitatively using a urinary albumin ELISA (Figure 6, B and C; see complete unedited blots in the supplemental material). The proteinuria was transient, peaking between 12 and 24 hours, and specific to IL-4, since transfer of antigen-specific B cells that were IL-4 deficient (IL-4 $4^{-/}$) did not induce proteinuria. IL- $4^{-/-}$Be 2 cells were only deficient in IL-4, and the other cytokines produced were made at the same levels as wildtype B cells (Supplemental Figure 4). Circulating IL-4 was undetectable by ELISA in the mice injected with antigen-specific B cells, suggesting that the effect was due to local B cell activation and release of IL-4 (data not shown). This was supported by 2-photon imaging of B cells in the kidney (37). 
A

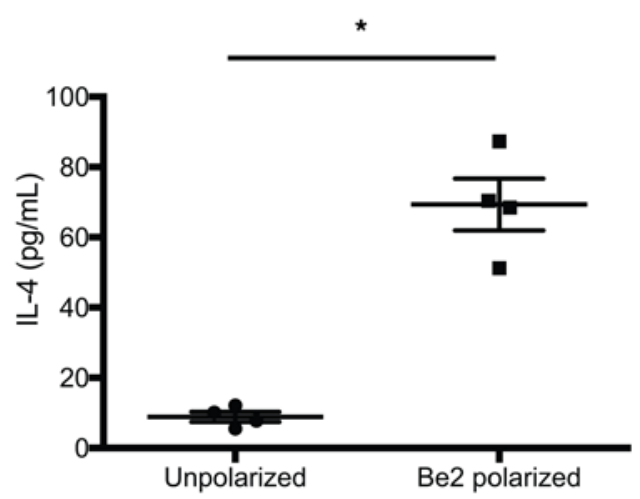

C

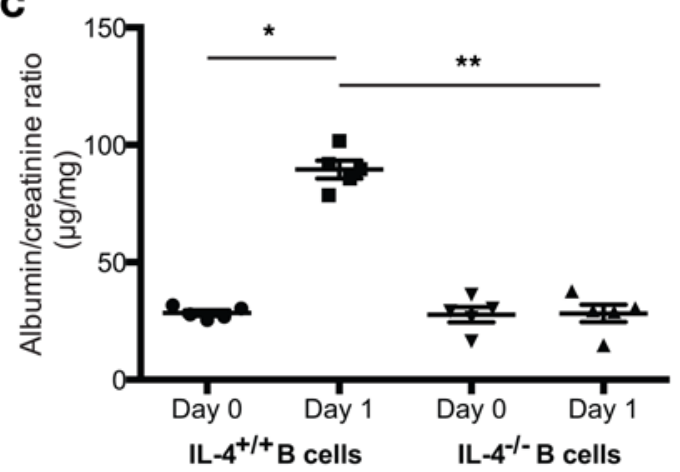

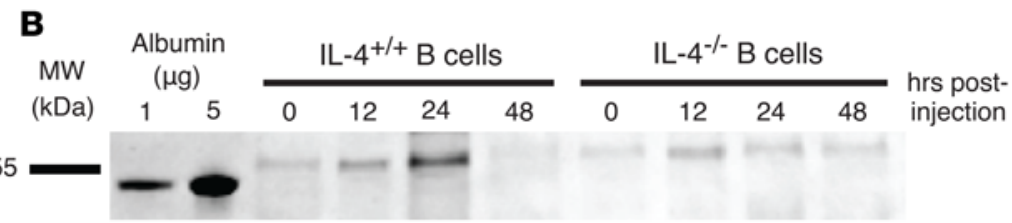

D

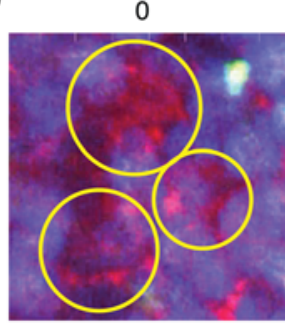

No
HEL

With

HEL

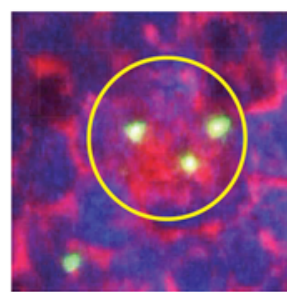

Minutes

2
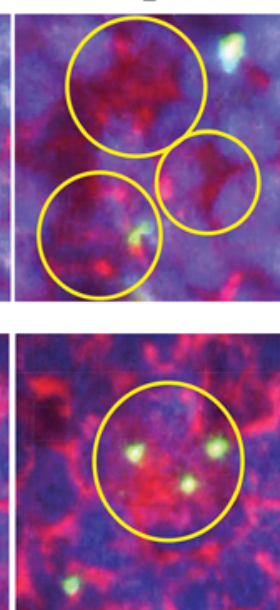

4
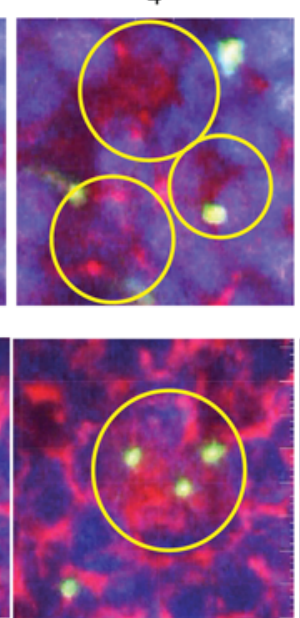
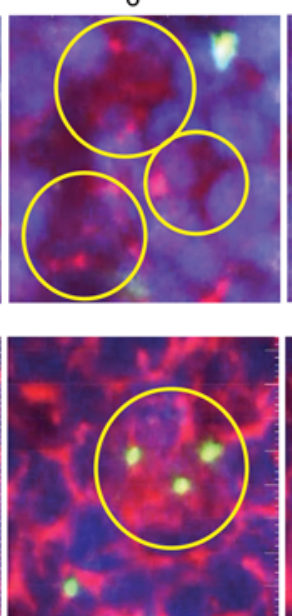

8
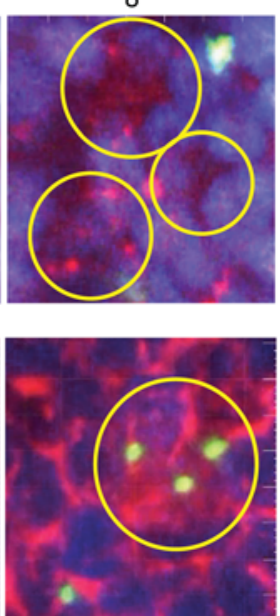

10
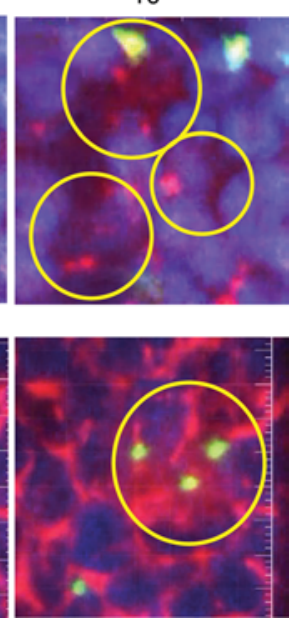

Figure 6. HEL-specific IL-4-secreting B cells generated proteinuria in mice treated with multimerized HEL. (A) HEL-specific B cells were either polarized in vitro into B effector 2 (Be2) cells to produce IL-4, or left unpolarized, and then stimulated through the B cell receptor with anti- $\mu$ antibody overnight. Only Be2-polarized B cells secreted IL-4 into the supernatant, as measured by ELISA. (B) Coomassie blue-stained SDS-PAGE and (C) spot albumin/creatinine ratios of urine from mice treated with multimerized HEL, followed by transfer of either Be2-polarized HEL-specific B cells or IL-4-deficient, Be2-polarized HEL-specific B cells. Proteinuria was observed only when B cells were capable of producing IL-4. Urine was collected 24 hours after B cell transfer. Symbols represent individual mice, and bars represent the mean. (D) Representative still images obtained from intravital 2-photon microscopy movies (Supplemental Videos 3 and 4) of mice injected with PBS (top) or multimerized HEL (bottom), followed by the transfer of fluorescently labeled HEL-specific B cells. When glomerular HEL was present, HEL-specific B cells arrested within glomeruli. In the absence of HEL, B cell trafficking through glomeruli was not changed. Quantum dots were injected i.v. to identify glomeruli and vessels. Glomeruli are outlined in circles. Red: glomeruli and vessels; blue: renal tubules; yellow: HEL-specific B cells. Original magnification, $\times 20$. Mean \pm SD of 2 experiments, with a total of 5 mice/group. ${ }^{*} P<0.001,{ }^{*} P<0.008$ by 2 -tailed Mann-Whitney.

After injection of antigen, mice were anesthetized and a kidney was exteriorized. Injection (i.v.) of fluorescent dextran labeled blood vessels and allowed us to identify glomeruli. In the absence of cognate antigen, labeled HEL-specific B cells trafficked rapidly through the glomerular capillaries. In contrast, when antigen was present, B cells entered the glomerular capillaries and arrested their movement within the glomeruli (Figure 6D and Supplemental Videos 3 and 4). B cell arrest within the glomerular capillaries when antigen was present suggests that in situ recognition of antigen leads to B cell activation and cytokine secretion.

Since the activation of B cells within glomeruli could induce inflammatory changes, we performed light and immunofluorescence microscopy to assess for pathologic changes. We observed no obvious morphologic alterations in the glomeruli of proteinuric mice by light microscopy at all time points tested 
A

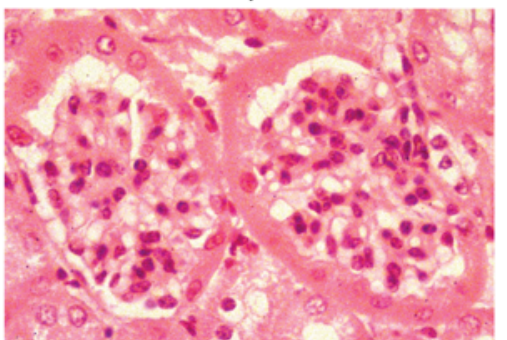

B
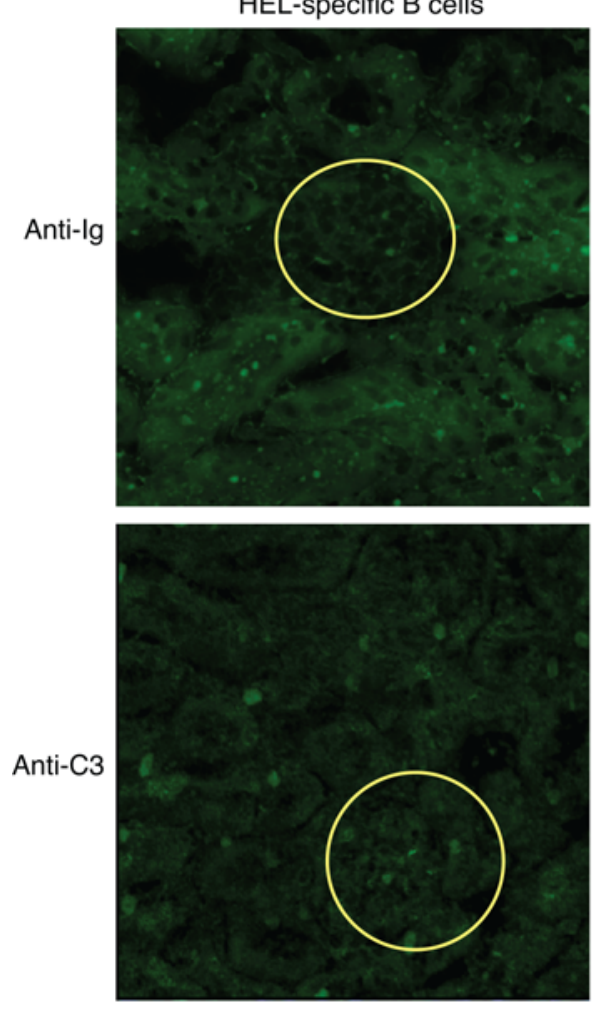

C

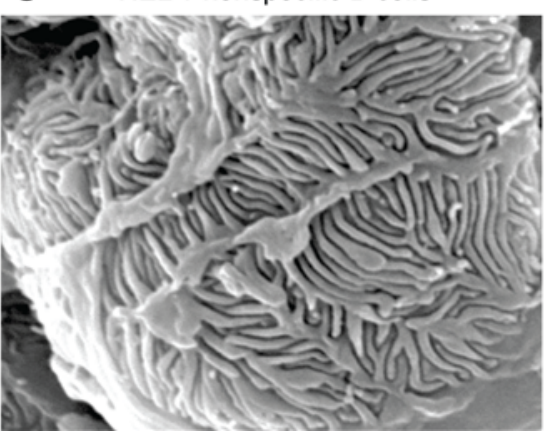

HEL + HEL-specific B cells

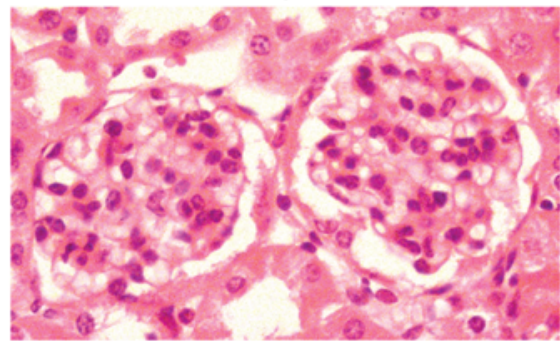

Positive Control
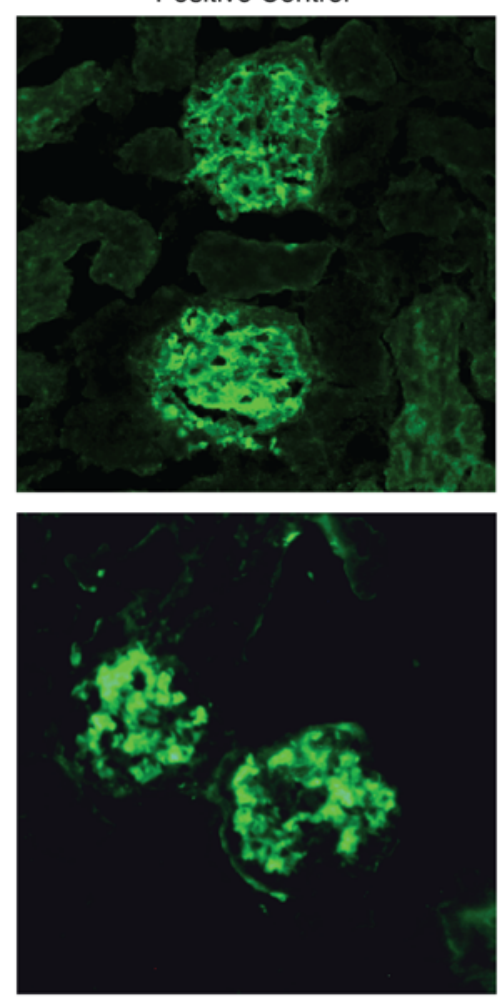

HEL + HEL-specific B cells

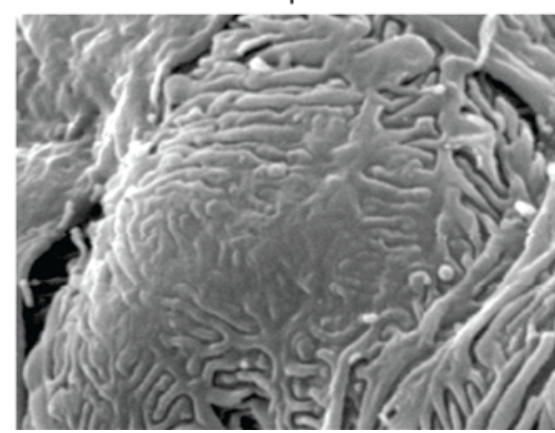

Figure 7. B cell-induced proteinuria generated foot process effacement. (A) Representative light microscopy of H\&E-stained kidney sections from mice with $B$ cell-induced proteinuria revealed no pathologic changes. Original magnification, $\times 40$. (B) Representative immunofluorescence microscopy images of kidneys from mice with B cell-induced proteinuria (left) did not demonstrate immunoglobulin or complement component $\mathrm{C} 3$ in glomeruli compared to positive control nephrotoxic serum (right). (C) Representative scanning electron microscopy from kidneys showed focal foot process effacement in mice with $B$ cell-induced proteinuria (right) compared with control mice (left). Scale bar: $10 \mu \mathrm{m}$. Data are representative of 3 independent experiments.

(Figure 7A). In addition, immunofluorescence staining of sections from proteinuric mice showed no detectable immunoglobulin or complement deposition in glomeruli (Figure 7B). Ultrastructural analysis of the glomeruli using scanning electron microscopy confirmed the presence of focal foot process effacement in proteinuric mice, while nonproteinuric control mice had normal foot processes (Figure 7C). Thus, B cells, activated in the kidney, can induce proteinuria and foot process effacement independent of antibodies and immune complexes.

Glomerular STAT6 activation occurs in a subset of MCD patients. STAT6 phosphorylation is specific for signaling by IL-4 and IL-13. To test if pSTAT6 can be detected in humans with nephrotic syndrome, we stained kidney biopsy sections from patients with MCD for STAT6 activation. Since most patients with MCD respond to initial treatment with glucocorticoids, patients are often biopsied only after treatment failure, limiting the availability of naive biopsy tissue. Such patients are also by definition steroid resistant and not likely to be sensitive to BCDTs. Twenty-nine patients with MCD, in which a biopsy was obtained before initiation of treatment, were identified from the Renal Pathology Services at Washington University School of Medicine, the University of Michigan, and King's College/Evelina Children's Hospital (Supplemental Table 1). For controls, we stained 23 kidney biopsies obtained for conditions unrelated to nephrotic syndrome (see Methods for diagnoses). Two of the twenty-nine MCD biopsies demonstrated strong glomerular pSTAT6 staining, and eight had weakly positive staining (see Methods for definitions). Of 23 controls, only one showed weakly positive pSTAT6 staining (Figure 8). While this is a small sample, these data support the hypothesis that IL-4 and/or IL-13 may be involved in some patients with MCD. 

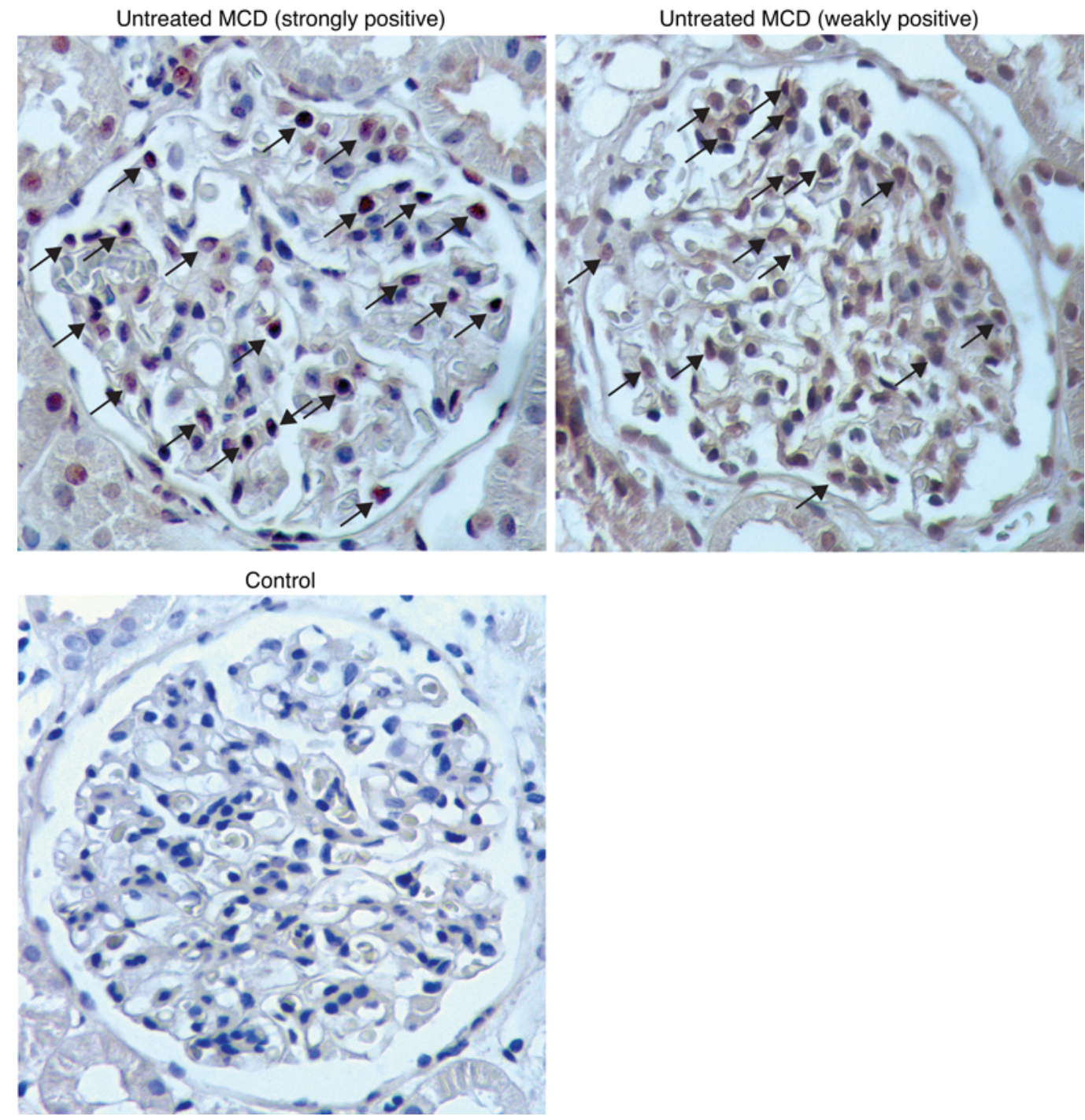

Figure 8. A subset of minimal change disease patients has activated glomerular STAT6. Representative immunohistochemistry of glomerular pSTAT6 expression in selected MCD patients (described in Supplemental Table 1) and a control. Ten of twenty-nine MCD patients screened demonstrated pSTAT6 staining (untreated MCD, arrows point to stained nuclei). Twenty-two of twenty-three healthy controls had no detectable pSTAT6 staining. Of 29 MCD patient samples stained for pSTAT6, 2 were strongly positive, 8 were weakly positive, and 19 were negative; of 22 controls samples stained for pSTAT6, 1 was weakly positive and 22 were negative $(P=0.019)$. Original magnification, $\times 400.1$

\section{Discussion}

Since the first reports of rituximab in the treatment of nephrotic diseases in 2004, its use has become an important part of the treatment armamentarium, especially for steroid-sensitive and relapsing forms of MCD and FSGS. Since there is no known role for B cells in these diseases, how and why rituximab mediates its efficacy is a mystery. One potential explanation is the ability of rituximab to directly bind to SMPDL3B on the podocyte (16), reversing changes in integrin affinity. Reports that ofatumumab, a CD20 antibody that binds to an epitope distinct from rituximab $(38,39)$, is also efficacious in patients with steroid-resistant FSGS (6) motivated us to reexamine this question. SMPDL3B, a protein expressed on podocytes but also broadly expressed on monocytes and gut epithelia, was initially proposed to bind to rituximab, as it contains a peptide sequence, in the reverse orientation, to an epitope identified in a screen for rituximab-binding sequences (40). Previous binding assays used fixed samples and amplification steps to visualize staining of podocytes with rituximab, indicating that the binding is weak (16). Our data suggest that the binding is not specific, suggesting that B cell depletion is the mechanism of action. 
Nephrotic diseases, such as MCD and FSGS, are by definition not caused by autoantibodies or by immune complexes. A major diagnostic feature for both MCD and FSGS is foot process effacement. The responsiveness of these diseases to immunosuppressive treatments such as glucocorticoids, calcineurin inhibitors, and mycophenolate has led many to consider these T cell-mediated diseases, even without specific evidence. The efficacy of BCDTs in the treatment of some fraction of cases, however, supports an immune component to these diseases.

In recent years, antibody-independent roles for B cells have emerged as important in several disease models. B cells express MHC class II and are excellent antigen-presenting cells. In mouse models of SLE, depletion of B cells impairs the activation of autoreactive $\mathrm{T}$ cells, suggesting that B cells play important roles as antigen-presenting cells (11). Recently, there has been a growing appreciation for the regulatory effects of B cell-derived cytokines, such as IL-10 and IL-35, on attenuating organ-specific autoimmunity in experimental autoimmune encephalitis $(14,15)$. B cells can also make a wide array of both Th1 and Th2 cytokines both in vitro $(41-45)$ and in vivo $(12,13)$.

We and others have suggested that an imbalance between the Rac and Rho G proteins underlies the process of foot process effacement $(24,25,46)$. Rho functions to maintain large actin structures important for cell adhesion, while Rac functions to stimulate cell migration and membrane ruffling and spreading $(25,26)$. During injury, Rac becomes active and induces the process of foot process effacement (24). Using this knowledge, we used markers of Rac activation, such as membrane ruffling in cultured podocytes, as a surrogate for foot process effacement. We confirmed the effects of IL-4 on podocytes in situ assay using an assay that we developed using minced renal cortices treated ex vivo with cytokines. Our success using these assays to identify IL-4 suggests that this assay could also be used to screen for the elusive soluble factors that have been speculated to cause nephrotic syndrome (47).

A pathogenic role for cytokine signaling in proteinuric diseases is supported by a recent publication describing a transgenic mouse overexpressing JAK2 in podocytes (48). When crossed with the Akita diabetic nephropathy mouse model infused with angiotensin II, enhanced proteinuria was observed that was largely reversed with JAK2 inhibition. As JAK2 is regulated by cytokines, these data help to link cytokines with foot process effacement.

The role of IL-4 in other glomerular injury models is controversial. It is implicated in the development of proteinuria in models of graft-versus-host disease (49-51), some models of SLE (52, 53), and IgA nephropathy (54-58) but attenuates proteinuria induced by autologous models of nephrotoxic nephritis (59-63). Given that autologous nephrotoxic nephritis relies on immunization against sheep antibody and that IL-4 has direct effects on antibody isotype switching, the role of IL-4 in nephrotoxic nephritis is complex and not solely dependent on its effect on podocytes.

We are not the first to propose a role of IL-4 and IL-13 in nephrotic syndrome. IL-4 is secreted by Th2 helper T cells and associated with atopy, a condition that is highly associated with MCD $(64,65)$. Transgenic mice expressing IL-4 develop glomerulosclerosis $(53,66-68)$ and transgenic rats expressing IL-13 develop proteinuria (69). While it was reported previously that podocytes express the IL-4 receptor (IL-4Ro and common $\gamma$-chain) and can respond to IL-4 (23), attempts to link IL-4 levels in serum and urine with nephrotic diseases have been largely unrevealing $(70,71)$. One possibility is that IL- 4 is produced locally at levels too low to be detected in blood or urine.

To test whether this was a viable hypothesis, we generated a model that would allow for B cell activation in the glomerulus. This suggested that activated B cells could produce enough IL-4 to induce proteinuria. Notably, we were unable to detect IL-4 in the serum, showing that local IL-4 can be pathogenic. While this may not be the mechanism of disease in humans, it does suggest other potential mechanisms. Recent data show that memory lymphocytes can exist as long-term residents of tissues such as lung and skin and far outnumber the number of lymphocytes circulating in the peripheral immune system (72). These cells often function as cytokine-secreting sentinel cells that respond to pathogens and/or antigens that transit through organs. Little is known yet about memory lymphocytes or innate lymphocytes in kidney, but these specialized cells could be relevant. Finally, polyclonal B cell activators, such as TLR ligands, released during infections and trapped by the glomerulus, could be another potent stimulus of B cell activation (73).

We noted that IL-13 was able to induce some level of membrane ruffling in our in vitro assay. While this is consistent with a previous study in rats showing that IL-13 can induce proteinuria, IL-13 plasmid administration in mice did not induce proteinuria (data not shown). The reason for the difference between 
mice and rats remains unknown but may represent species-specific requirements for cytokine-induced proteinuria. Furthermore, the role of IL-13 in SSNS patients remains unclear (74), as glucocorticoid treatment increases serum IL-13 (75).

We directly assessed for IL-4 signaling in kidney biopsies from MCD patients. Biopsies from untreated MCD patients are difficult to procure, as patients are often initially treated without biopsy, particularly in the United States. Despite this, we identified 29 kidney biopsies of untreated MCD patients, of which 10 had either strongly or weakly positive glomerular staining for activated STAT6. Antibody staining of formalin-fixed paraffin-embedded tissue can be challenging, and we were careful to score only for strong nuclear staining in glomerular nuclei. This was clear-cut in two of our samples, as blindly evaluated by three pathologists independently. In some of the samples ( 8 of 29 patients, 1 of 22 controls), glomerular nuclear staining was detected, along with weak cytoplasmic staining in glomerular and/or tubular cells. While the cytoplasmic staining likely represents nonspecific background staining, we categorized these samples in a separate group. Importantly, both groups of patients with pSTAT6 staining responded to steroids, consistent with an immune mechanism of disease.

Most of our samples did not show pSTAT6, supporting the heterogeneity of MCD, which may have other causes (76-78), such as CLCF1 (47) or ANGPTL4 (78). Additionally, while 90\% of MCD patients respond to glucocorticoids (79), most of these patients will experience a relapse requiring additional therapy $(79,80)$. The identification of a subset of patients with MCD due to IL-4 may lead to more personalized and specific therapeutic approaches for the treatment of SSNS. Our work also potentially explains the association of atopy with MCD as well as the efficacy of steroids and rituximab in some patients.

\section{Methods}

Reagents. Anti-SMPDL3B polyclonal antibody was purchased from Genway (catalog GWB-2281D4). Anti-HA (catalog 901501, clone 16B12) antibody, Alexa Fluor 488-conjugated anti-HA (catalog 901509, clone 16B12) antibody, Alexa Fluor 488 IgG1, k isotype control (catalog 400132), and Alexa Fluor 647 mouse IgG2a, k isotype control (catalog 400234) were purchased from Biolegend. Anti-CD20 polyclonal antibody (catalog PA5-16701) was purchased from Thermo Fisher Scientific. Anti- $\beta$-actin antibody (cata$\log$ A1978, clone AC-15) was purchased from MilliporeSigma. Rituximab was produced in-house by the Antibody Engineering Department at Genentech (81). Alexa Fluor 647-conjugated rituximab was generated using the Alexa Fluor 647 Antibody Labeling Kit (Thermo Fisher Scientific). Recombinant murine IL-13 and TNF- $\alpha$ (Peprotech) and recombinant murine EGF (Cell Signaling Technology) were reconstituted using the manufacturer's recommendations. Recombinant murine IL-4, IFN- $\gamma$, and anti-mouse IL-4 monoclonal antibody (clone 11B11) (82) were gifts from Robert Schreiber, Washington University in St. Louis. FITC-conjugated anti-HEL monoclonal antibody (clone F10.6.6; Emil Unanue, Washington University School of Medicine) (83) and nephrotoxic serum (Jeffrey Kopp, National Institute of Diabetes and Digestive and Kidney Diseases, NIH, Bethesda, Maryland, USA) were also provided. FITC-conjugated polyclonal anti-mouse C3 F(ab')2 fragment (catalog 0855510) was purchased from MP Biomedicals. AntipSTAT6 (pTyr641, catalog SAB4300038) polyclonal antibody was purchased from MilliporeSigma. All secondary antibodies used purchased from Jackson ImmunoResearch Laboratories Inc.

Mice. Mice were maintained under specific pathogen-free conditions in the Washington University School of Medicine animal facilities according to institutional animal care guidelines. 129X1/SvJ (catalog 000691), B6.129S2-Ighm ${ }^{\text {tmlCgn}} / \mathrm{J}$ (muMT) (84) (catalog 0002288), and B6.129P2-IL4 ${ }^{\text {tmlCgn }} / \mathrm{J}$ (IL-4-deficient) (85) (catalog 0002253) mice were purchased from Jackson Laboratory. HELmuMT (MD4 line) mice (86) (originally purchased from Jackson Laboratory, catalog 002595) were provided by Emil Unanue. IL-4-deficient HELmuMT mice were generated in two steps: (a) crossing IL-4-knockout mice to muMT mice to produce B cell-deficient, IL-4-deficient mice, and (b) crossing IL-4-deficient, muMT mice with HELmuMT to produce IL-4-deficient mice with HEL-transgenic B cells. All mice used were 6- to 8-week-old females.

Generation of HA-tagged SMPDL3B construct. A mammalian expression construct of human SMPDL3B (pCMV6-XL5 human SMPDL3B transcript variant 1, NM_014474) was purchased from Origene. HA-tag (YPYDVPDYA) was inserted downstream of the signal peptide (aa 1-18) using the QuikChange II XL site-directed mutagenesis kit (Agilent) according to the manufacturer's instructions.

Cell culture and transient transfection. 293T cells (catalog CRL-3216, ATCC) and HeLa cells (catalog CCL-2, ATCC) were maintained at $37^{\circ} \mathrm{C}$ in DMEM supplemented with $10 \%$ FBS. 293T cells and HeLa cells were transfected using Lipofectamine 2000 and Lipofectamine 3000 (ThermoFisher), respectively, in 
6-well plates at approximately $70 \%$ confluency. Conditionally immortalized murine podocyte cell line generation and propagation have been previously described $(87,88)$. Podocytes were propagated at $33^{\circ} \mathrm{C}$ (permissive temperature) on collagen-I-coated dishes in RPMI, 10\% FBS, and $10 \mathrm{U} / \mathrm{ml}$ recombinant mouse IFN- $\gamma$. Differentiation was induced by changing media to RPMI, 5\% FBS without IFN- $\gamma$, and shifting the cells to $37^{\circ} \mathrm{C}$ (nonpermissive temperature) for at least 7 days. After differentiation, cells arrested their growth, increased size, and developed elongated cell processes.

Western blot. Cells were lysed in a modified RIPA lysis buffer (50 mM Tris- $\mathrm{HCl}$ [pH 7.4], $150 \mathrm{mM} \mathrm{NaCl}, 5$ mM EDTA, $1 \%$ Triton X-100, 0.5\% sodium deoxycholate, cOmplete mini protease inhibitor cocktail [Roche]) for 30 minutes on ice. Samples were separated by SDS-PAGE, transferred to nitrocellulose membranes, and immunoblotted for SMPDL3B, HA, CD20, and $\beta$-actin. The blots were visualized by infrared imaging on an Odyssey Imaging System (LI-COR Biosciences). To remove glycan groups, samples were treated with PNGase F (New England Biolabs) according to the manufacturer's instructions prior to SDS-PAGE.

Flow cytometry. The cells were washed with ice-cold PBS and dissociated using a nonenzymatic cell dissociation buffer (Thermo Fisher Scientific). For nonfixed cells, cells were directly resuspended in FACS buffer (2\% FBS and 2 mM EDTA in $1 \times$ PBS). For fixed cells, cells were incubated in 4\% PFA (Electron Microscopy Sciences) in PBS ( $\mathrm{pH}$ 7.4) for 15 minutes at room temperature, washed 3 times in cold PBS, and then resuspended in FACS buffer. Cells were incubated with Alexa Fluor 488-conjugated anti-HA antibody $(0.5 \mu \mathrm{g} / \mathrm{ml})$ and Alexa Fluor 647 -conjugated rituximab $(5 \mu \mathrm{g} / \mathrm{ml})$ for 30 minutes at $4^{\circ} \mathrm{C}$. FACS data were collected on a BD LSRFortessa flow cytometer (BD Biosciences) and analyzed with FlowJo software (FlowJo).

Light and immunofluorescence microscopy. To confirm membrane localization of HA-SMPDL3B, 293T cells were plated and transfected in Nunc Lab-Tek II CC2 chamber slides (Thermo Fisher Scientific). Cells were fixed with 4\% PFA in PBS for 15 minutes at room temperature, washed, and stained with Alexa Fluor 488-conjugated anti-HA antibody $(0.5 \mu \mathrm{g} / \mathrm{ml})$ overnight at $4^{\circ} \mathrm{C}$. Coverslips were mounted with ProLong Gold Antifade Mountant with DAPI (Thermo Fisher Scientific), and images were captured with a Nikon A1R confocal microscope. For H\&E staining, kidneys were fixed with 10\% buffered formalin solution and embedded in paraffin. $5-\mu \mathrm{m}$ sections were collected and then stained with H\&E. Otherwise, kidneys were snap frozen in OCT media and $7-\mu \mathrm{m}$ sections were collected. For immunofluorescence, sections were stained with primary antibody for 1 hour at room temperature (anti-HEL $=1: 250$, anti-Ig $=1: 200$, anti-C3 = 1:200) and treated with the appropriate secondary antibody. Some sections were counterstained with Hoechst dye to visualize nuclei. Coverslips were mounted with ProLong Antifade mounting medium (Invitrogen), and images were captured with an Olympus FluoView FV1000 microscope. For immunohistochemistry, mice were anesthetized and then perfused with 4\% PFA. Kidneys were cryoprotected with sucrose solution. Deidentified sections from human kidney biopsies were obtained from the Kidney Translational Research Core at Washington University in St. Louis, the University of Michigan, and Guy's \& St. Thomas' Hospital (London, United Kingdom). The diagnosis of MCD was confirmed by the absence of light and immunofluorescence pathology and the presence of diffuse foot process effacement by transmission electron microscopy. Control patients included those with the following diagnoses: tubulointerstitial nephritis, acute kidney injury in the absence of glomerular pathology, clean margins from kidneys resected for renal cell carcinoma, and transplanted donor kidney at the time of transplant into recipient (off of immunosuppression). Formalin-fixed paraffin-embedded human kidney biopsy sections were subject to tris-EDTA antigen retrieval prior to immunostaining. Frozen sections were fixed in $4 \%$ PFA. Kidney sections were stained with primary antibody (anti-pSTAT6 $=1: 100$ ) overnight at $+4^{\circ} \mathrm{C}$, followed by incubation with secondary antibody. Sections were developed using the DAB Substrate Kit (Thermo Scientific). Counterstaining was performed with hematoxylin and $10 \mathrm{mM}$ sodium bicarbonate solution. Sections were dehydrated and mounted with VectaMount (Vector Laboratories). Images were obtained using a Nikon Eclipse E800 microscope.

Membrane ruffling assay and kymograph analysis. Murine podocytes were cultured in glass-bottomed dishes at $37^{\circ} \mathrm{C}$ for 10 days to allow for full differentiation. Eight hours prior to imaging, the cells were washed and cultured in serum-free media to arrest any baseline membrane ruffling. Sequential images were captured by an Olympus FluoView FV1000 microscope every 10 seconds for a 20-minute duration prior to and after cytokine addition. Anti-IL-4 antibody and JAK inhibitor were added to podocytes 1 hour prior to IL-4 addition. Olympus Fluoview software was used to assemble movies. The ImageJ plug-in, Multiple Kymograph (NIH; http://rsbweb.nih.gov/ij), was used to create kymo- 
graphs at 5 different locations of maximum membrane ruffling for each cell imaged (89). Ten actin spikes were measured for each kymograph, and average length (ruffling index) was determined as previously described (27).

Scanning electron microscopy. For visualizing foot processes in minced renal cortices, kidneys were harvested from 129X1/SvJ mice and placed in media composed of RPMI/F12, $15 \mathrm{mM} \mathrm{HEPES,} 10$

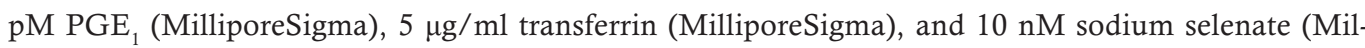
liporeSigma). The cortex of the kidney was minced using sterile blades and cultured in media with or without cytokine or antibody for 20 minutes at $37^{\circ} \mathrm{C}$. The tissue was washed in PBS and then fixed in $0.2 \mathrm{M}$ sodium cacodylate buffer (Electron Microscopy Services) with 2\% PFA and 2\% glutaraldehyde. Samples were post-fixed in 1\% phosphate-buffered osmium tetroxide, dehydrated in graded ethanols, and critical-point dried in carbon dioxide. The samples were mounted onto stubs and then sputter-coated using gold/palladium. For all other scanning electron microscopy images, kidneys were harvested and cortices were diced and processed as described above. Fifteen glomeruli per sample were analyzed using a Hitachi S-2600H scanning electron microscope at the Microscopy and Digital Imaging Core at Washington University in St. Louis.

Assessment of pSTAT6 staining. The stratification of pSTAT6 staining (negative, weakly positive, and strongly positive) was based on these criteria: negative - no nuclear staining or no enhanced nuclear staining over background (cytoplasmic); weakly positive - enhanced nuclear staining noticeable over background; and strongly positive - pronounced nuclear staining over background. Two blinded pathologists scored all slides for the presence of pSTAT6 staining.

Intravital 2-photon microscopy. The preparation of kidney intravital 2-photon microscopy was adapted from previously described approaches (90-92). Briefly, 8- to 10-week-old mice previously given multimerized HEL were anesthetized with isofluorane and a small vertical incision was made in the flank of the mouse. The kidney was exposed, and an upper chamber plate painted with a thin ring of VetBond tissue adhesive $(3 \mathrm{M})$ was gently lowered on to the kidney to secure it to the cover glass and seal the peritoneal cavity. A dual-channel heating system (Warner Instruments) warmed the imaging chamber to $37^{\circ} \mathrm{C}$. CFSE-labeled HEL-specific B cells were then transferred by tail vein injection, followed with $2,000 \mathrm{kD}$ Rhodamine dextran to label vessels and glomeruli. Time-lapse imaging was performed using a custom-built 2-photon microscope and ImageWarp acquisition software (A\&B Software). Images were acquired either at video rate $(30 \mathrm{f} / \mathrm{sec})$ or by time-lapse imaging, where we averaged 15 video-rate frames $(0.5 \mathrm{~s} /$ slice $)$ creating a $Z$-stack of 31 sequential $Z$-steps $(2.5 \mu \mathrm{m}$ each). Each optical section was approximately $220 \times 240 \mu \mathrm{m}$ in the $x$ and $y$ dimensions.

IL-4 piggyBac plasmid construction, hydrodynamic injection, and tofacitinib administration. Plasmid encoding murine IL-4 was a gift from Ken Murphy (Washington University in St. Louis). IL-4 was amplified and cloned into the PB530A-2 vector (System Biosciences) using BamHI and NotI. The piggyBac/IL-4 and transposase vectors were prepped using the Qiagen EndoFree Plasmid Maxi Kit and combined at a 5:1 ratio (total DNA $=45 \mu \mathrm{g}$ ) in approximately $2 \mathrm{ml}$ TransIT QR Hydrodynamic Delivery Solution (Mirus Bio). Hydrodynamic injection of these vectors occurred through the tail vein using a 30-gauge needle. Serum IL-4 was determined using the Mouse IL-4 Quantikine ELISA Kit (R\&D Systems). For the JAK inhibitor experiments, tofacitinib (Selleckchem) was mixed with $0.5 \%$ methylcellulose and $0.025 \%$ Tween-20 in water, and $50 \mathrm{mg} / \mathrm{kg}$ was administered by oral gavage to mice twice daily.

Assessment of proteinuria. For SDS-PAGE analysis, $5 \mu 1$ urine was loaded with $\times 4$ Laemmli sample buffer onto a 4\%-20\% SDS polyacrylamide gel (Bio-Rad). Proteins were visualized by staining the gel with Coomassie blue. For quantification, the Mouse Albumin ELISA Quantification Set (Bethyl Laboratories Inc.) and QuantiChrom Creatinine Assay Kit (Bioassay Systems) were used using manufacturer's directions.

Production of multimerized HEL and injection into mice. HEL (Amresco) was dissolved in sterile PBS (Hyclone) and treated with NHS-biotin (Thermo Scientific), followed with dialysis using Slide-A-Lyzer Dialysis Cassettes G2 (Thermo Scientific) against sterile PBS. Avidin (Invitrogen) was slowly added to the solution of biotinylated HEL to generate multimerized HEL. The size of multimerized HEL was confirmed using SDS-PAGE, followed by Coomassie blue staining. Multimerized HEL (1 mg) was delivered i.v. via tail vein injection into mice, and organs were harvested 30 minutes to 7 days after infusion.

HEL-specific B cell isolation, polarization, CFSE labeling, and adoptive transfer into mice. Spleens from HELmuMT or IL-4-deficient HELmuMT mice were collected into a cell strainer (100- $\mu \mathrm{m}$ nylon mesh) and mashed through. Splenocytes were washed and resuspended in PBS supplemented with $2 \%$ FBS 
and 2 mM EDTA. B cells were enriched by negative isolation using the Dynabeads Mouse CD43 (Untouched B cells) Isolation Kit (Invitrogen). B cell purity was validated by flow cytometry (using B220 as the B cell marker), and consistently was $>95 \%$. Unpolarized B cells were polarized into IL-4secreting B cells as previously described $(13,36)$. Purified HEL-specific B cells were CFSE-labeled per the manufacturer's instructions (CellTrace CFSE Cell Proliferation Kit, Molecular Probes/Invitrogen). $5 \times 10^{6} \mathrm{~B}$ cells were transferred via tail vein injection into wild-type hosts previously administered multimerized HEL.

Statistics. All data, except for pSTAT6 characterization data, are represented as mean \pm SD. For membrane ruffling results, group differences were assessed by repeated-measures 1-way ANOVA with Bonferroni correction. For pSTAT6 characterization, Fisher's exact test was used. For all other results, 2-tailed Mann-Whitney test was used. A $P$ value of less than 0.05 was considered significant.

Study approval. All animal experiments were conducted with approval from the Washington University in St. Louis Animal Care and Use Committee (St. Louis, Missouri, USA). Patient samples were obtained in a deidentified manner, with institutional review board approval from King's College/Evelina Children's Hospital, the Kidney Translational Research Core (Washington University in St. Louis), and the University of Michigan.

\section{Author contributions}

AHJK designed and performed the experiments, analyzed the experiments, and wrote the manuscript. JJC performed the SMPDL3B experiments and wrote the manuscript. SA designed the experiments, performed the blinded evaluation of pSTAT6 staining on human kidney sections, and edited the manuscript. AK, SJ, and JBH provided human kidney sections. MJM designed and performed the 2-photon microscopy experiments. TSS performed the blinded evaluation of pSTAT6 staining on human kidney sections and edited the manuscript. JHM designed the experiments and edited the manuscript. ASS designed the experiments, participated in data analysis, and wrote and edited the manuscript.

\section{Acknowledgments}

This work was supported by grants from the Rheumatology Research Foundation (Scientist Development Award and Investigator Award) to AHJK; from the NIH (K08DK088944) and the NephCure-ASN Foundation to JBH; from the NIH (R01DK058366) to ASS; and the Howard Hughes Medical Institute. Patient recruitment and specimen services were supported by the Washington University in St. Louis George M. O'Brien Center for Kidney Translational Research Core (NIH P30 DK079333) and the Renal Division at Washington University in St. Louis. Electron microscopy work was supported in part by the Washington University in St. Louis Microscopy and Digital Imaging Core (NIH P30 DC004665). We appreciate the efforts of Emil Unanue and Ta-Chiang Liu in assisting with the blinded scoring of pSTAT6 staining in the MCD patient slides. We are grateful for the services of the Washington University in St. Louis Kidney Translational Research Core (specifically Kristen Aubuchon and Mary Hoffman for patient recruitment and Amanda Knoten for technical assistance with histology sections from patient biopsies). We acknowledge Yu Tao in the Washington University in St. Louis Research Design and Biostatistics Group for the Institute of Clinical and Translational Sciences for statistical assistance. We thank Jaclynn Lett for electron microscopy sample preparation in the Microscopy and Digital Imaging Core in the Research Center for Auditory and Visual Studies, Department of Otolaryngology, Washington University School of Medicine. We also thank Facundo Batista for assistance with HEL multimerization.

Address correspondence to: Andrey S. Shaw, Genentech, One DNA Way, South San Francisco, California 94080, USA. Phone: 650.225.2367; Email: shaw.andrey@gene.com.

SA's present address is: Department of Pathology, University of Washington, Seattle, Washington, USA

MJM's present address is: Division of Infectious Disease, Department of Internal Medicine, Washington University School of Medicine, St. Louis, Missouri, USA.

JJC and ASS's present address is: Genentech, South San Francisco, California, USA. 
1. Chan AC. B cell immunotherapy in autoimmunity--2010 update. Mol Immunol. 2011;48(11):1344-1347.

2. Martin F, Chan AC. B cell immunobiology in disease: evolving concepts from the clinic. Annu Rev Immunol. 2006;24:467-496.

3. Townsend MJ, Monroe JG, Chan AC. B-cell targeted therapies in human autoimmune diseases: an updated perspective. Immunol Rev. 2010;237(1):264-283.

4. Iijima $\mathrm{K}$, et al. Rituximab for childhood-onset, complicated, frequently relapsing nephrotic syndrome or steroid-dependent nephrotic syndrome: a multicentre, double-blind, randomised, placebo-controlled trial. Lancet. 2014;384(9950):1273-1281.

5. Sinha A, Bagga A. Rituximab therapy in nephrotic syndrome: implications for patients' management. Nat Rev Nephrol. 2013;9(3):154-169.

6. Basu B. Ofatumumab for rituximab-resistant nephrotic syndrome. N Engl J Med. 2014;370(13):1268-1270.

7. Schnaper HW, Robson AA, Kopp JB. Nephrotic syndrome: Minimal change nephropathy, focal segmental glomerulosclerosis, and collapsing glomerulopathy. Diseases of the Kidney and Urinary Tract. 8th ed. Schrier RW (ed). Philadelphia: Lippincott Williams and Wilkins; 2007: p.1585.

8. Shalhoub RJ. Pathogenesis of lipoid nephrosis: a disorder of T-cell function. Lancet. 1974;2(7880):556-560.

9. Savin VJ, et al. Circulating factor associated with increased glomerular permeability to albumin in recurrent focal segmental glomerulosclerosis. N Engl J Med. 1996;334(14):878-883.

10. Dantal J, et al. Effect of plasma protein adsorption on protein excretion in kidney-transplant recipients with recurrent nephrotic syndrome. N Engl J Med. 1994;330(1):7-14.

11. Chan O, Shlomchik MJ. A new role for B cells in systemic autoimmunity: B cells promote spontaneous T cell activation in MRL-lpr/lpr mice. J Immunol. 1998;160(1):51-59.

12. Bermejo DA, et al. Trypanosoma cruzi trans-sialidase initiates a program independent of the transcription factors ROR $\gamma \mathrm{t}$ and Ahr that leads to IL-17 production by activated B cells. Nat Immunol. 2013;14(5):514-522.

13. Harris DP, et al. Reciprocal regulation of polarized cytokine production by effector B and T cells. Nat Immunol. 2000;1(6):475-482

14. Fillatreau S, Sweenie CH, McGeachy MJ, Gray D, Anderton SM. B cells regulate autoimmunity by provision of IL-10. Nat Immunol. 2002;3(10):944-950.

15. Shen P, et al. IL-35-producing B cells are critical regulators of immunity during autoimmune and infectious diseases. Nature. 2014;507(7492):366-370.

16. Fornoni A, et al. Rituximab targets podocytes in recurrent focal segmental glomerulosclerosis. Sci Transl Med. 2011;3(85):85ra46.

17. Duddy ME, Alter A, Bar-Or A. Distinct profiles of human B cell effector cytokines: a role in immune regulation? J Immunol. 2004;172(6):3422-3427.

18. Johansson-Lindbom B, Borrebaeck CA. Germinal center B cells constitute a predominant physiological source of IL-4: implication for Th2 development in vivo. J Immunol. 2002;168(7):3165-3172.

19. Liu Q, et al. The role of B cells in the development of CD4 effector T cells during a polarized Th2 immune response. J Immunol. 2007;179(6):3821-3830

20. Shen P, Fillatreau S. Antibody-independent functions of B cells: a focus on cytokines. Nat Rev Immunol. 2015;15(7):441-451.

21. Bruggeman LA, Drawz PE, Kahoud N, Lin K, Barisoni L, Nelson PJ. TNFR2 interposes the proliferative and NF- $\mathrm{kB}-\mathrm{mediated}$ inflammatory response by podocytes to TNF- $\alpha$. Lab Invest. 2011;91(3):413-425.

22. Baudeau C, et al. Induction of MHC class II molecules HLA-DR, -DP and -DQ and ICAM 1 in human podocytes by gamma-interferon. Exp Nephrol. 1994;2(5):306-312.

23. Van Den Berg JG, et al. Interleukin-4 and interleukin-13 act on glomerular visceral epithelial cells. J Am Soc Nephrol. 2000;11(3):413-422.

24. Yu H, et al. Racl activation in podocytes induces rapid foot process effacement and proteinuria. Mol Cell Biol. 2013;33(23):4755-4764

25. Akilesh S, et al. Arhgap24 inactivates Rac1 in mouse podocytes, and a mutant form is associated with familial focal segmental glomerulosclerosis. J Clin Invest. 2011;121(10):4127-4137.

26. Hall A. Rho GTPases and the actin cytoskeleton. Science. 1998;279(5350):509-514.

27. Moeller MJ, et al. Protocadherin FAT1 binds Ena/VASP proteins and is necessary for actin dynamics and cell polarization. EMBO J. 2004;23(19):3769-3779.

28. Ridley AJ, Paterson HF, Johnston CL, Diekmann D, Hall A. The small GTP-binding protein rac regulates growth factor-induced membrane ruffling. Cell. 1992;70(3):401-410.

29. Ding S, Wu X, Li G, Han M, Zhuang Y, Xu T. Efficient transposition of the piggyBac (PB) transposon in mammalian cells and mice. Cell. 2005;122(3):473-483.

30. Nakanishi H, Higuchi Y, Kawakami S, Yamashita F, Hashida M. piggyBac transposon-mediated long-term gene expression in mice. Mol Ther. 2010;18(4):707-714.

31. Saridey SK, et al. PiggyBac transposon-based inducible gene expression in vivo after somatic cell gene transfer. Mol Ther. 2009;17(12):2115-2120.

32. Andrianaivo F, Lecocq M, Wattiaux-De Coninck S, Wattiaux R, Jadot M. Hydrodynamics-based transfection of the liver: entrance into hepatocytes of DNA that causes expression takes place very early after injection. J Gene Med. 2004;6(8):877-883

33. Nelms K, Keegan AD, Zamorano J, Ryan JJ, Paul WE. The IL-4 receptor: signaling mechanisms and biologic functions. Annu Rev Immunol. 1999;17:701-738.

34. Fleischmann R, et al. Placebo-controlled trial of tofacitinib monotherapy in rheumatoid arthritis. N Engl J Med. 2012;367(6):495-507.

35. Goodnow CC, et al. Altered immunoglobulin expression and functional silencing of self-reactive B lymphocytes in transgenic mice. Nature. 1988;334(6184):676-682.

36. Harris DP, Goodrich S, Mohrs K, Mohrs M, Lund FE. Cutting edge: the development of IL-4-producing B cells (B effector 2 cells) is controlled by IL-4, IL-4 receptor alpha, and Th2 cells. J Immunol. 2005;175(11):7103-7107.

37. Cahalan MD, Parker I, Wei SH, Miller MJ. Two-photon tissue imaging: seeing the immune system in a fresh light. Nat Rev Immunol. 2002;2(11):872-880. 
38. Cheson BD. Ofatumumab, a novel anti-CD20 monoclonal antibody for the treatment of B-cell malignancies. J Clin Oncol. 2010;28(21):3525-3530.

39. Teeling JL, et al. The biological activity of human CD20 monoclonal antibodies is linked to unique epitopes on CD20. J Immunol. 2006;177(1):362-371.

40. Perosa F, Favoino E, Caragnano MA, Dammacco F. Generation of biologically active linear and cyclic peptides has revealed a unique fine specificity of rituximab and its possible cross-reactivity with acid sphingomyelinase-like phosphodiesterase $3 \mathrm{~b}$ precursor. Blood. 2006;107(3):1070-1077.

41. Kindler V, Matthes T, Jeannin P, Zubler RH. Interleukin-2 secretion by human B lymphocytes occurs as a late event and requires additional stimulation after CD40 cross-linking. Eur J Immunol. 1995;25(5):1239-1243.

42. O'Garra A, et al. Production of cytokines by mouse B cells: B lymphomas and normal B cells produce interleukin 10. Int Immunol. 1990;2(9):821-832.

43. Ohnishi E, Iwata T, Inouye S, Kurata T, Sairenji T. Interleukin-4 production in Epstein-Barr virus-transformed B cell lines from peripheral mononuclear cells of patients with atopic dermatitis. J Interferon Cytokine Res. 1997;17(10):597-602.

44. Pistoia V. Production of cytokines by human B cells in health and disease. Immunol Today. 1997;18(7):343-350

45. Schultze JL, et al. Human non-germinal center B cell interleukin (IL)-12 production is primarily regulated by $\mathrm{T}$ cell signals CD40 ligand, interferon gamma, and IL-10: role of B cells in the maintenance of T cell responses. J Exp Med. 1999;189(1):1-12.

46. Mundel P, Reiser J. Proteinuria: an enzymatic disease of the podocyte? Kidney Int. 2010;77(7):571-580.

47. McCarthy ET, Sharma M, Savin VJ. Circulating permeability factors in idiopathic nephrotic syndrome and focal segmental glomerulosclerosis. Clin J Am Soc Nephrol. 2010;5(11):2115-2121.

48. Zhang H, et al. Podocyte-specific JAK2 overexpression worsens diabetic kidney disease in mice. Kidney Int. 2017;92(4):909-921

49. Meyers CM, Tomaszewski JE, Glass JD, Chen CW. The nephritogenic T cell response in murine chronic graft-versus-host disease. J Immunol. 1998;161(10):5321-5330.

50. Ellison CA, Gibson IW, Hayglass KT, Gartner JG. Effect of palifermin in a murine model of graft-versus-host disease (GVHD) associated with Th2 cytokine production, autoantibody production, and glomerulonephritis. J Clin Immunol. 2006;26(5):485-494.

51. Ellison CA, Bradley DS, Fischer JM, Hayglass KT, Gartner JG. Murine graft-versus-host disease induced using interferongamma-deficient grafts features antibodies to double-stranded DNA, T helper 2-type cytokines and hypereosinophilia. Immunology. 2002;105(1):63-72.

52. Peng SL, Moslehi J, Craft J. Roles of interferon-gamma and interleukin-4 in murine lupus. J Clin Invest. 1997;99(8):1936-1946.

53. Erb KJ, Rüger B, von Brevern M, Ryffel B, Schimpl A, Rivett K. Constitutive expression of interleukin (IL)-4 in vivo causes autoimmune-type disorders in mice. J Exp Med. 1997;185(2):329-339.

54. Chintalacharuvu SR, et al. T cell cytokine polarity as a determinant of immunoglobulin A (IgA) glycosylation and the severity of experimental IgA nephropathy. Clin Exp Immunol. 2008;153(3):456-462.

55. Ebihara I, Hirayama K, Yamamoto S, Muro K, Yamagata K, Koyama A. Th2 predominance at the single-cell level in patients with IgA nephropathy. Nephrol Dial Transplant. 2001;16(9):1783-1789.

56. Lai KN, Ho RT, Lai CK, Chan CH, Li PK. Increase of both circulating Th1 and Th2 T lymphocyte subsets in IgA nephropathy. Clin Exp Immunol. 1994;96(1):116-121.

57. Scivittaro V, et al. Profiles of immunoregulatory cytokine production in vitro in patients with IgA nephropathy and their kindred. Clin Exp Immunol. 1994;96(2):311-316.

58. Ballardie FW, Gordon MT, Sharpe PT, Darvill AM, Cheng H. Intrarenal cytokine mRNA expression and location in normal and IgA nephropathy tissue: TGF alpha, TGF beta, IGF 1, IL-4 and IL-6. Nephrol Dial Transplant. 1994;9(11):1545-1552.

59. Cook HT, Singh SJ, Wembridge DE, Smith J, Tam FW, Pusey CD. Interleukin-4 ameliorates crescentic glomerulonephritis in Wistar Kyoto rats. Kidney Int. 1999;55(4):1319-1326.

60. Kitching AR, Tipping PG, Huang XR, Mutch DA, Holdsworth SR. Interleukin-4 and interleukin-10 attenuate established crescentic glomerulonephritis in mice. Kidney Int. 1997;52(1):52-59.

61. Kitching AR, Tipping PG, Mutch DA, Huang XR, Holdsworth SR. Interleukin-4 deficiency enhances Th1 responses and crescentic glomerulonephritis in mice. Kidney Int. 1998;53(1):112-118.

62. Kluth DC, et al. Macrophages transfected with adenovirus to express IL-4 reduce inflammation in experimental glomerulonephritis. J Immunol. 2001;166(7):4728-4736.

63. Tipping PG, Kitching AR, Huang XR, Mutch DA, Holdsworth SR. Immune modulation with interleukin-4 and interleukin-10 prevents crescent formation and glomerular injury in experimental glomerulonephritis. Eur J Immunol. 1997;27(2):530-537.

64. Abdel-Hafez M, Shimada M, Lee PY, Johnson RJ, Garin EH. Idiopathic nephrotic syndrome and atopy: is there a common link? Am J Kidney Dis. 2009;54(5):945-953.

65. Mathieson PW. Immune dysregulation in minimal change nephropathy. Nephrol Dial Transplant. 2003;18 Suppl 6:vi26-vi29.

66. Foote LC, et al. Interleukin-4 produces a breakdown of tolerance in vivo with autoantibody formation and tissue damage. Autoimmunity. 2004;37(8):569-577.

67. Rüger BM, Erb KJ, He Y, Lane JM, Davis PF, Hasan Q. Interleukin-4 transgenic mice develop glomerulosclerosis independent of immunoglobulin deposition. Eur J Immunol. 2000;30(9):2698-2703.

68. Rüger BM, Hasan Q, Erb KJ, Davis PF. Progression of renal disease in interleukin-4 transgenic mice: involvement of transforming growth factor-beta. Int J Exp Pathol. 1999;80(3):113-123.

69. Lai KW, et al. Overexpression of interleukin-13 induces minimal-change-like nephropathy in rats. J Am Soc Nephrol. 2007;18(5):1476-1485.

70. Youn YS, Lim HH, Lee JH. The clinical characteristics of steroid responsive nephrotic syndrome of children according to the serum immunoglobulin E levels and cytokines. Yonsei Med J. 2012;53(4):715-722.

71. Araya CE, et al. A case of unfulfilled expectations. Cytokines in idiopathic minimal lesion nephrotic syndrome. Pediatr Nephrol. 2006;21(5):603-610

72. Fan X, Rudensky AY. Hallmarks of tissue-resident lymphocytes. Cell. 2016;164(6):1198-1211.

73. Rawlings DJ, Schwartz MA, Jackson SW, Meyer-Bahlburg A. Integration of B cell responses through Toll-like receptors and antigen receptors. Nat Rev Immunol. 2012;12(4):282-294. 
74. Yap HK, Cheung W, Murugasu B, Sim SK, Seah CC, Jordan SC. Th1 and Th2 cytokine mRNA profiles in childhood nephrotic syndrome: evidence for increased IL-13 mRNA expression in relapse. J Am Soc Nephrol. 1999;10(3):529-537.

75. Tain YL, Chen TY, Yang KD. Implications of serum TNF-beta and IL-13 in the treatment response of childhood nephrotic syndrome. Cytokine. 2003;21(3):155-159.

76. Delville M, et al. A circulating antibody panel for pretransplant prediction of FSGS recurrence after kidney transplantation. Sci Transl Med. 2014;6(256):256ra136.

77. Wei C, et al. Circulating urokinase receptor as a cause of focal segmental glomerulosclerosis. Nat Med. 2011;17(8):952-960.

78. Clement LC, et al. Podocyte-secreted angiopoietin-like-4 mediates proteinuria in glucocorticoid-sensitive nephrotic syndrome. Nat Med. 2011;17(1):117-122.

79. Tarshish P, Tobin JN, Bernstein J, Edelmann CM. Prognostic significance of the early course of minimal change nephrotic syndrome: report of the International Study of Kidney Disease in Children. J Am Soc Nephrol. 1997;8(5):769-776.

80. Hodson EM, Willis NS, Craig JC. Corticosteroid therapy for nephrotic syndrome in children. Cochrane Database Syst Rev. 2007;(4):CD001533.

81. Reff ME, et al. Depletion of B cells in vivo by a chimeric mouse human monoclonal antibody to CD20. Blood. 1994;83(2):435-445.

82. Ohara J, Paul WE. Production of a monoclonal antibody to and molecular characterization of B-cell stimulatory factor-1. Nature. 1985;315(6017):333-336.

83. Fischmann T, Souchon H, Riottot MM, Tello D, Poljak RJ. Crystallization and preliminary x-ray diffraction studies of two new antigen-antibody (lysozyme-Fab) complexes. J Mol Biol. 1988;203(2):527-529.

84. Kitamura D, Roes J, Kühn R, Rajewsky K. A B cell-deficient mouse by targeted disruption of the membrane exon of the immunoglobulin mu chain gene. Nature. 1991;350(6317):423-426.

85. Kühn R, Rajewsky K, Müller W. Generation and analysis of interleukin-4 deficient mice. Science. 1991;254(5032):707-710.

86. Mason DY, Jones M, Goodnow CC. Development and follicular localization of tolerant B lymphocytes in lysozyme/anti-lysozyme IgM/IgD transgenic mice. Int Immunol. 1992;4(2):163-175.

87. Akilesh S, et al. Podocytes use FcRn to clear IgG from the glomerular basement membrane. Proc Natl Acad Sci USA 2008;105(3):967-972.

88. Mundel $\mathrm{P}$, et al. Rearrangements of the cytoskeleton and cell contacts induce process formation during differentiation of conditionally immortalized mouse podocyte cell lines. Exp Cell Res. 1997;236(1):248-258.

89. Abramoff MD, Magelhaes PJ, Ram SJ. Image processing with ImageJ. Biophotonics International. 2004;11:36-42.

90. Camirand G, et al. Multiphoton intravital microscopy of the transplanted mouse kidney. Am J Transplant. 2011;11(10):2067-2074

91. Devi S, et al. Multiphoton imaging reveals a new leukocyte recruitment paradigm in the glomerulus. Nat Med. 2013;19(1):107-112.

92. Yang JJ, Kularatne SA, Chen X, Low PS, Wang E. Characterization of in vivo disulfide-reduction mediated drug release in mouse kidneys. Mol Pharm. 2012;9(2):310-317. 